\title{
Einige Reaktionen des Chlordioxyds und der chlorigen Säure.
}

\author{
Von \\ Wilutam Bray.
}

Eine eingehende Untersuchung der Chlorsauerstoffverbindungen, ${ }^{1}$ besonders des $\mathrm{ClO}_{2}$, führte zum Ergebnis, dafs viele Reaktionen dieses Stoffes unter dem einheitlichen Gesichtspunkt betrachtet werden können, dals primär aus $\mathrm{ClO}_{2}$ bei der Reduktion das Chlorition $\mathrm{ClO}_{2}^{\prime}$, bezw. die chlorige Säure $\mathrm{ClO}_{2} \mathrm{H}$ entsteht. Eine Reihe anderer Reaktionen liefs sich bisher nicht so einfach deuten; dies noch nicht abgerundete Beobachtungsmaterial ist aber in verschiedenen Hinsichten interessant genug, um eine Veröffentlichung desselben berechtigt erscheinen zu lassen.

Was die Gewinnung und die Eigenschaften des $\mathrm{ClO}_{2}$ betrifft, so sei auf die schon erwähnte ausführliche Mitteilung hingewiesen, ein gleiches gilt vom historischen Rückblick. Nur die analytischen Methoden mögen auch an dieser Stelle kurz beschrieben werden, da sie zum Verständnis des Folgenden unumgänglich notwendig sind.

\section{\& I. Analytische Methoden.}

$\mathrm{Da}$ das $\mathrm{ClO}_{2}$ in wässeriger Lösung einen beträchtlichen Dampfdruck besitzt und daher beim Aufsaugen in die Pipette stark verdunstet, so sind die obersten Schichten stets zu verdünnt. $\mathrm{Um}$ die Fehler, die bei konzentrierten Lösungen hierdurch bedingt werden, $\mathrm{zu}$ vermeiden, wurden Pipetten verwandt, wie sie $\mathrm{J}_{\text {AKOWKIN }}{ }^{2}$ empfohlen hat, nämlich solche, bei denen die verdünnten oberen Schichten durch einen Dreiweghahn in der Pipette zurückgehalten werden. Die Titration der konzentrierten Lösungen wurde mit einer kleinen,

1 Zeitschr. phys. Chem. 54 (1906) (unter Druck).

2 Zeitschr. phys. Chem. 29 (1899), 626. 
rasch ausfliefsenden Pipette dieser Art ausgeführt, deren Inhalt unterbalb des Hahnes $2.62 \mathrm{ccm}$ betrug. Beim Entleeren wurde die Spitze der Pipette in die Flüssigkeit getaucht, und nach dem Ausfliefsen wurde sie (unterhalb des Hahnes) mit Wasser nachgespült. Wegen letzteren Verfahrens und der Kleinheit der Pipette wurde sie als Aufnahmepipette mit Quecksilber kalibriert.

Die Stoffe, deren Bestimmung in Frage kam, waren $\mathrm{ClO}_{2}, \mathrm{Cl}_{2}$, $\mathrm{ClO}_{3}{ }^{\prime}, \mathrm{ClO}_{2}{ }^{\prime}, \mathrm{ClO}^{\prime}, \mathrm{Cl}^{\prime}, \mathrm{OH}^{\prime}$ und $\mathrm{H}$. Von diesen kommt in Lösungen, die $\mathrm{HCl}$ enthalten - und um solche handelt es sich bei den Ausgangslösungen immer $-\mathrm{ClO}^{\prime}$ nicht in Betracht, da es mit $\mathrm{HCl}$ schnell nach der Gleichung:

reagiert.

$$
\mathrm{HClO}+\mathrm{HCl}=\mathrm{Cl}_{2}+\mathrm{H}_{2} \mathrm{O}
$$

Wie es mit alkalischen Lösungen steht, in denen Hypochloritbildung zu berücksichtigen ist, wird an Ort und Stelle besprochen werden.

$\mathrm{ClO}_{2}$ und $\mathrm{Cl}_{2}$ konnten durch ihre Flüchtigkeit von den anderen Stoffen getrennt werden. Es wurde so verfahren, dafs ein Stickstoff- (oder Luft-)strom durch die zu analysierende Lösung und dann durch eine Glasrohrleitung in eine neutrale Jodkaliumlösung geleitet wurde. Da durch den Gasstrom ausgeschiedenes $J$ mitgenommen werden konnte, war eine zweite mit JK gefüllte Flasche hinter die erste geschaltet, die etwas Thiosulfat enthielt. Man mufs immer kleine Mengen der Lösung auf ihren Gehalt an $\mathrm{ClO}_{2}$ und $\mathrm{Cl}_{2}$ prüfen, und die Gase durch den Luftstrom vollständig entfernen, weil man sonst wegen der verschiedenen Flüchtigkeit der beiden Gase falsche Werte erhält.

$\mathrm{ClO}_{2}$ und $\mathrm{Cl}_{2}$ lassen sich am besten derart nebeneinander bestimmen, dafs man ihr Gemisch einmal in saurer, das andere Mal in neutraler Lösung mit JK reagieren lälst. Die Bestimmung des in saurer Lösung abgeschiedenen Jods, die als "Jodtitration" bezeichnet wird, wurde in der Weise ausgeführt, dafs zu $50 \mathrm{ccm}$ einer $0.1-0.2$ normalen Jodkaliumlösung zunächst eine gemessene Menge der fraglichen Lösung und hierauf $3-5 \mathrm{ccm}$ 2-normaler Schwefelsäure hinzugefügt wurde. Nach drei Minuten wurde dann das ausgeschiedene Jod mit Thiosulfat (meist mit 0.1 norm.) titriert. Das Warten war nötig, weil die letzten Stadien der Reaktion nicht unmelsbar rasch verliefen und weil beim allzu frübzeitigen Hinzufügen des Thiosulfats ein Teil desselben zu Sulfat oxydiert werden würde. 
Vermeidet man diese Fehler, so lassen sich $\mathrm{ClO}_{2}$-Lösungen sicher bestimmen, unabhängig von der Konzentration der benutzten Reagentien und von der Mischungsfolge. Es verläuft also die Reaktion:

$$
\mathrm{ClO}_{2}+5 \mathrm{~J}^{\prime}+4 \mathrm{H}^{\cdot}=5 \mathrm{~J}+\mathrm{Cl}^{\prime}+2 \mathrm{H}_{2} \mathrm{O}
$$

anscheinend ohne von störenden Nebenreaktionen begleitet zu sein.

Die Bestimmung des $\mathrm{ClO}_{2}$ neben $\mathrm{Cl}_{2}$ beruht nun darauf, dals in neutraler Lösung $\mathrm{ClO}_{2}$ nur ungefähr ein Fünftel von dem Jod in Freiheit setzt, das in saurer Lösung abgeschieden wird. Während $\mathrm{Cl}_{2}$ in beiden Fällen nach der Gleichung:

$$
\mathrm{Cl}_{2}+2 \mathrm{~J}^{\prime}=2 \mathrm{Cl}^{\prime}+\mathrm{J}_{2}
$$

reagiert, reagiert $\mathrm{ClO}_{2}$ in neutraler Lösung nach der Gleichung:

$$
3 \mathrm{ClO}_{2}+5 \mathrm{~J}^{\prime}=2 \mathrm{JO}_{3}{ }^{\prime}+3 \mathrm{Cl}^{\prime}+3 \mathrm{~J} .
$$

Die „Jodtitration" verhält sich also zur "Jodtitration neutral“" für $\mathrm{ClO}_{2}$ angenähert wie $5: 1$; genauer ergab sich $4.8: 1$; für $\mathrm{Cl}$ wie $1: 1$. Aus den beiden Gleichungen:

$$
\begin{aligned}
4.8\left(\mathrm{ClO}_{2}\right)+(\mathrm{Cl}) & =\text { Jodtitration } \\
\left(\mathrm{ClO}_{2}\right)+(\mathrm{Cl}) & =\text { Jodtitration neutral }
\end{aligned}
$$

konnte die Konzentration der beiden Stoffe bestimmt werden, und zwar ist hier die Konzentration des $\mathrm{ClO}_{2}$ in Molekülen, die des $\mathrm{Cl}_{2}$ in g-Atomen im Liter gerechnet.

In einigen wenigen Fällen wurde statt dieses umständlichen Verfahrens einfach die auf $\mathrm{ClO}_{2}$ und $\mathrm{Cl}_{2}$ zu untersuchende Lösung zu einer verdünnten neutralen JK-Lösung gegeben und unmittelbar danach Natriumbikarbonat zugesetzt, um die Reaktion zwischen $\mathrm{JO}_{3} \mathrm{H}$ und $\mathrm{J}^{\prime} \mathrm{zu}$ hemmen. Diese Methode ist nicht zu empfehlen, da die Reaktion $\mathrm{JO}_{3} \mathrm{H}+\mathrm{J}^{\prime}$ sehr rasch verläuft. Statt der Verhältniszahl 4.8 mufste wegen des so hervorgerufenen Fehlers eine kleinere (4.6-4.7) benutzt werden. Es war nicht zu umgehen, dafs die Bikarbonatlösung erst nach der Zugabe der $\mathrm{ClO}_{2}+\mathrm{Cl}_{2}$-haltigen Flüssigkeit zugegossen wurde, man durfte nicht etwa die letzte Lösung zu einer bikarbonathaltigen JK-Lösung fügen, sonst wirkt $\mathrm{Cl}_{2}$ auf $\mathrm{J}^{\prime}$ unter $\mathrm{JO}_{3}^{\prime}$-Bildung ein, ${ }^{1}$ während bei nachträglichem $\mathrm{Zu}$ satz des Bikarbonats, das normal mit dem JK reagierende $\mathrm{Cl}_{2}$ schon völlig $\mathrm{zu} \mathrm{Cl}$ reduziert worden ist.

1 Zeitschr. phys. Chem. 54 (1906) 463. 
Abgesehen von diesen beiden flüchtigen Stoffen kommen noch $\mathrm{ClO}_{3}{ }^{\prime}, \mathrm{ClO}_{2}{ }^{\prime}, \mathrm{Cl}^{\prime}$ und $\mathrm{H} \cdot$ für die Analyse in Betracht. $\mathrm{ClO}_{2}^{\prime}$ ergibt sich durch die Jodtitration; es zeigte sich, dals auch der Vorgang:

$$
\mathrm{ClO}_{2}{ }^{\prime}+4 \mathrm{~J}^{\prime}+4 \mathrm{H}^{\cdot}=4 \mathrm{~J}+\mathrm{Cl}^{\prime}+2 \mathrm{H}_{2} \mathrm{O}
$$

ohne störende Nebenvorgänge verläuft.

Zur Bestimmung des $\mathrm{ClO}_{3}{ }^{\prime}$ diente die „Ferrosulfattitration", die sowohl für reine Chlorsäurelösungen, wie für Gemische $\mathrm{HClO}_{3}+$ $\mathrm{ClO}_{2}+\mathrm{Cl}_{2}$, bezw. $\mathrm{ClO}_{3}{ }^{\prime}+\mathrm{ClO}_{2}{ }^{\prime}+\mathrm{ClO}^{\prime}$ in Anwendung kam. Falls es sich un reine Chlorsäurelösungen handelte, wurde die $\mathrm{HClO}_{3}$ mit überschüssigem Ferrosulfat + konzentrierter Schwefelsäure (unter Kohlensäure) einige Minuten bei $100^{\circ}$ behandelt. Nach dem $\mathbf{A b}$ kühlen unter $\mathrm{CO}_{2}$ wurde das überschüssige Ferrosulfat in der üblichen Weise mit Permanganat titriert. In den anderen Fällen wurden die Lösungen bei Zimmertemperatur gemischt (um Verlust an $\mathrm{ClO}_{2} \mathrm{zu}$ vermeiden) und dann wie oben behandelt. Die Reaktion zwischen $\mathrm{ClO}_{2}$ und Ferrosulfat verläuft praktisch momentan, ist aber für die Bestimmung von $\mathrm{ClO}_{2}$ nicht brauchbar, weil $\mathrm{ClO}_{3} \mathrm{H}$ dabei entsteht. ${ }^{1}$

Die Differenz der Ferrosulfat- und der Jodtitration ergab die Chlorsäurekonzentration; hatte man das $\mathrm{ClO}_{2}\left(\mathrm{ev} .+\mathrm{Cl}_{2}\right)$ mittels eines Luftstroms entfernt, so ergab die Ferrosulfattitration unmittelbar die $\mathrm{ClO}_{3} \mathrm{H}-\mathrm{K}$ onzentration. Schliefslich konnte man letztere auch dadurch feststellen, dal's man die $\mathrm{Cl}^{\prime}$-Konzentration von der $\mathrm{H}^{\prime}$-Konzentration abzog. Erstere wurde mit $\mathrm{AgNO}_{3}$ (mit $\mathrm{K}_{2} \mathrm{CrO}_{4}$ als Indikator) in neutraler Lösung bestimmt; verdünnte Säuren wurden mit Barytwasser, konzentrierte mit kohlensäurefreier Natronlauge titriert. Die Versuche lehrten, dafs die Säuretitration (nach Entfernen des $\mathrm{ClO}_{2}$ und $\mathrm{Cl}_{2}$ ) gleich der Summe der $\mathrm{Cl}^{\prime}$ - und der Ferrosulfattitration war, ein Zeichen, dals nur diese zwei Säuren, $\mathrm{HCl}$ und $\mathrm{HClO}_{3}$, vorhanden waren. Dies war vor allem auch deshalb wichtig, weil es Reaktionen des $\mathrm{ClO}_{2}$ (z. B. die Zersetzung) zu verfolgen erlaubte, ohne dafs man auf Bestimmung der Oxydationswerte zu verschiedenen Zeiten angewiesen gewesen wäre; letzteres Verfahren bätte wegen der Flüchtigkeit des $\mathrm{ClO}_{2}$ zu ungenauen Ergebnissen geführt.

Was weitere Einzelheiten (Beleganalysen usw.) betrifft, so muls bezüglich dieser auf die schon erwähnte Abhandlung ${ }^{2}$ verwiesen werden.

' Zeitschr. phys. Chem. 54 (1906) (unter Druck).

${ }^{2}$ Zeitschr. phys. Chem. 54 (1906) (unter Druck). 


\section{§ II. Uber die Zersetzung von $\mathbf{C l O}_{2}$-Lösungen.}

\section{Zersetzung einer reinen $\mathrm{ClO}_{2}$-Lösung.}

Es wurde gleich bei den ersten Versuchen mit $\mathrm{ClO}_{2}$ gefunden, dafs die Lösungen desselben nicht so unbeständig waren, wie früher angenommen worden ist. Die Zersetzung wird bekanntlich durch Licht und Wärme beschleunigt. Ich habe die Zersetzungsgeschwindigkeit bei $0^{0}$ im Dunkeln gemessen, und babe ferner den Einflufs der Reaktionsprodukte und verschiedener Katalysatoren bestimmt. Die $\mathrm{ClO}_{2}$-Lösung wurde in zugeschmolzenen Rohren aufbewahrt und nach der gew ünschten Zeit in der oben beschriebenen Weise auf die verschiedenen möglichen Stoffe untersucht. Es stellte sich heraus, dals die Zersetzungsgeschwindigkeit einer reinen $\mathrm{ClO}_{2}$-Lösung praktisch gleich Null ist, wie folgende Versuche zeigen. Die Anfangskonzentration des Chlordioxyds und der SalzsäureVerunreinigung, und das Verhältnis der entstandenen $\mathrm{HClO}_{3} \mathrm{zu}$ der entstandenen $\mathrm{HCl},\left(\frac{\mathrm{HClO}_{3}}{\mathrm{HCl}}\right)^{\text {" }}$ sind in der Tabelle angegeben.

\section{Tabelle 1.}

Zersetzung bei $0^{0}$ im Dunkeln.

\begin{tabular}{|c|c|c|c|c|}
\hline $\begin{array}{l}\stackrel{\mathrm{ClO}_{\mathbf{2}^{-}}}{\text {Konzentr. }} \\
\text { in Molen }\end{array}$ & $\begin{array}{c}\text { HCl- } \\
\text { Konzentr. } \\
\text { in Molen }\end{array}$ & $\begin{array}{c}\text { Zeit } \\
\text { in } \\
\text { Wochen }\end{array}$ & $\begin{array}{c}\text { Zer- } \\
\text { setzung } \\
\text { in } \%\end{array}$ & $\begin{array}{c}\text { Entstandene } \\
-\frac{\mathrm{HClO}_{3}}{\mathrm{H}} \overline{\mathrm{Cl}}\end{array}$ \\
\hline 0.155 & 0.0011 & 7 & 0.6 & 20 \\
\hline 0.114 & 0.0010 & 5 & 3.4 & 3.0 \\
\hline 0.222 & 0.0020 & 6 & 2 & 2.2 \\
\hline
\end{tabular}

Diese langsame Zersetzung wurde auch für einige andere Lösungen beobachtet. Das Mittel für das Verhältnis $\left(\frac{\mathrm{HClO}_{3}}{\mathrm{HCl}}\right)$ war etwa 3.0. Der niedrige Wert dieses Verhältnisses und der unregelmälsige Verlauf der Zersetzung zeigten, dafs andere als die an anderer Stelle ${ }^{1}$ beschriebene Reaktion:

$$
6 \mathrm{ClO}_{2}+3 \mathrm{H}_{2} \mathrm{O}=5 \mathrm{HClO}_{3}+\mathrm{HCl},
$$

welche durch Chlorion beschleunigt wurde, stattfinden.

Der Zweck der folgenden Versuche war, jede Reaktion für sich zu isolieren und zu untersuchen.

1 Zeitschr. phys. Chem. 54 (1906) (unter Druck).

Z. anorg. Chem. Bd. 48. 


\section{Der Einflufs der Reaktionsprodukte.}

Wie schon angegeben, wird die Zersetzung durch Chlorion beschleunigt und erfolgt bei Überschufs von $\mathrm{Cl}^{\prime}$ quantitativ nach der Gleichung:

$$
6 \mathrm{ClO}_{2}+3 \mathrm{H}_{2} \mathrm{O}=5 \mathrm{HClO}_{3}+\mathrm{HCl}^{1}
$$

Die Anwesenheit von Kaliumchlorat hat nur wenig Einflufs auf die Zersetzungsgeschwindigkeit. In einer $0.28 \mathrm{Mol} . \mathrm{ClO}_{2}$ Lösung, welche 0.167 Mol. in bezug auf $\mathrm{ClO}_{3}{ }^{\prime}$ und $0.0014 \mathrm{Mol}$. auf $\mathrm{HCl}$ war, hatte sich in fünf Wochen nur $3.8 \%$ zersetzt.

Das Verhältnis $\left(\frac{\mathrm{HClO}_{3}}{\mathrm{HCl}}\right)$ war 2.7.

Der Einflufs des H-Ions ist praktisch null. In einer Probe der Lösung (a), Tabelle 1, welche $1 / 40$ normal in bezug auf Schwefelsäure war, batte sich in sieben Wochen nur $0.8 \%$ zersetzt $\left(\frac{\mathrm{HClO}_{3}}{\mathrm{HCl}}=2.0\right)$.

Die Anwesenheit von Chlor beschleunigt die Zersetzung. Für nicht zu kleine Mengen von Chlor wurde das Verhältnis $\left(\frac{\mathrm{HClO}_{3}}{\mathrm{HCl}}\right)$ annähernd = 1 gefunden. Die Untersuchungen werde ich weiter unten beschreiben. Die Reaktion findet nach dem Schema statt:

$$
\mathrm{ClO}_{2}+1 / 2 \mathrm{Cl}_{2}+\mathrm{H}_{2} \mathrm{O}=\mathrm{HClO}_{3}+\mathrm{HCl} \text {. }
$$

Die Untersuchung der Reaktion zwischen $\mathrm{HClO}_{3}$ und $\mathrm{HCl}$ zeigte, dafs die Reaktion eine umkehrbare ist $(\S \mathrm{V})$.

\section{Einflufs von platiniertem Platin.}

Bei Anwesenheit von platiniertem Platin findet dieselbe Reaktion statt, wie bei Anwesenheit von Chlorion; nämlich:

$$
6 \mathrm{ClO}_{2}+3 \mathrm{H}_{2} \mathrm{O}=5 \mathrm{HClO}_{3}+\mathrm{HCl} \text {. }
$$

$50 \mathrm{ccm}$ einer $0.122 \mathrm{Mol}$, nach der ReYchlerschen ${ }^{2}$ Methode hergestellten $\mathrm{ClO}_{2}$-Lösung - diese sind stets etwas chlor-

${ }^{1}$ Zeitschr. phys. Chem. 54 (1906) (unter Druck).

${ }^{2}$ Bull. soc. chim. 25 (1901), 659. Diese $\mathrm{ClO}_{2}$-Lösungen werden durch Einwirkung von Kaliumchlorat auf Schwefelsäure hergestellt und enthalten, wie an anderer Stelle (Zeitschr. phys. Chem. 54 (1906) (unter Druck) gezeigt wurde, immer $\mathrm{Cl}_{2}$. So oft im folgenden Lösungen dieser Art benutzt wurden, ist dies durch ein $R$ gekennzeichnet worden. 
haltig - wurden in Berührung mit einem Stück platinierten Platin von $2 \mathrm{ccm}$ Oberfläche in 5 Monaten im Dunkeln bei Zimmertemperatur vollständig zersetzt. Fine Sauerstoffbildung wurde nicht beobachtet. Das Verhältnis $\frac{\mathrm{HClO}_{3}}{\mathrm{HCl}}$ war $=4.18$. Dieser niedrige Wert (anstatt 5) beruht offenbar auf der Anwesenheit von Chlor in der Lösung. Die Reaktion:

$$
3 \mathrm{Cl}_{2}+\mathrm{H}_{2} \mathrm{O}=\mathrm{HClO}_{3}+5 \mathrm{HCl}
$$

wird ebenfalls durch $\mathrm{Pt}^{1}$ und $\mathrm{ClO}_{2}$ (s.w. u.) beschleunigt, und da die resultierende Lösung kein $\mathrm{Cl}_{2}$ mehr enthielt, mulste die Umwandlung nach diesem Schema eine praktisch vollständige gewesen sein. Hierdurch wird das Verhältnis $\frac{\mathrm{HClO}_{3}}{\mathrm{HCl}}$ verringert und zwar braucht man nur eine Chlorverunreinigung von 4 Verbindungsgewichten Chlor auf $100 \mathrm{Mol}$. $\mathrm{ClO}_{2}$ anzunehmen, damit das Verhältnis $\frac{\mathrm{HClO}_{3}}{\mathrm{HCl}}$ von 5 auf 4.20 sinkt; in den nach der Methode von Rerchler hergestellten Lösungen kann sogar noch mehr Chlor enthalten sein. Es darf deshalb als sehr wahrscheinlich angesehen werden, dafs eine reine Lösung in Gegenwart von Platin nach der oben angegebenen Gleichung sich zersetzen wird.

\section{Einfluls von Kupfersulfat und Eisenalaun.}

Zusätze von $\mathrm{CuSO}_{4}$ und von Eisenalaun hatten kleine beschleunigende Wirkungen. Die Zersetzung ist ebenso wie die gewöhnliche (Tabelle 1) keine stöchiometrische.

\section{Einfluls der Temperatur.}

Die Zersetzungsgeschwindigkeit nimmt zu mit steigender Temperatur. Sie ist unregelmälsig und wurde nur in bezug auf Sauerstoffbildung untersucht. Diese wurde in mefsbarem Betrage bei $60^{\circ}$ (im Dunkeln) in zwei Tagen nicht beobachtet, wohl aber entstand viel Sauerstoff bei $100^{\circ}$; es folgt daraus, dafs die Reaktion, in welcher Sauerstoff gebildet wird, einen sehr grofsen Temperaturkoeffizienten besitzt. Folgende Versuche zeigen, dafs diese Reaktion eine einfache Zersetzung in Chlor und Sauerstoff ist.

${ }^{1}$ Sirk, Zeitschr. f. Elektroch. 11 (1905), 261. 


\section{Die Reaktion $\mathrm{ClO}_{2}=1 / 2 \mathrm{Cl}_{2}+\mathbf{0}_{2}$.}

Die Reaktion findet, wie oben erwähnt, im Dunkeln bei $100^{\circ}$ rasch statt. Chlorsäure und Salzsäure werden auch gebildet; aus dem Verlauf der Reaktion konnte leicht bewiesen werden, dafs diese Säuren in Folgereaktionen entstehen und dals die primäre Reaktion die Zersetzung in Chlor und Sauerstoff war.

Die Versuche wurden in zugeschmolzenen Röhren ausgeführt und die Konzentrationen der Säuren in der Weise bestimmt, wie es in $\S I$ beschrieben ist. Der Sauerstoff ist nicht gemessen worden; beim Öffnen des Rohres entwichen immer etwas Chlordioxyd und Chlor. Das Verhältnis zwischen Chlordioxyd und Chlor in der Lösung (nach Abkühlen) wurde aus dem Verhältnis der beiden Jodtitrationen annähernd festgestellt. Zur Kontrolle wurde die ursprüngliche $\mathrm{ClO}_{2}$-Konzentration aus den analytischen Resultaten berechnet. Wegen des Verlustes an $\mathrm{ClO}_{2}$ und Chlor mufste diese berechnete Konzentration immer kleiner sein als die theoretische. Das Gegenteil würde bedeuten, dals eine zu hohe Konzentration für Chlor angenommen worden war.

Um die etwas komplizierte Berechnung der Versuchsergebnisse klar zu machen, ist sie für den ersten Versuch im einzelnen angegeben.

In der Tabelle sind alle Konzentrationen als Mole pro Liter angeführt, nur der Wert der Jodtitration ist in Oxydationswerten notiert, da durch sie zwei Stoffe $\left(\mathrm{Cl}_{2}\right.$ und $\left.\mathrm{ClO}_{2}\right)$ bestimmt werden. Die Gesamtsäurekonzentration, die deshalb weniger genau war, weil die Säure mit dem Glas reagierte, wurde nur zur Kontrolle der Einzelbestimmungen des $\mathrm{Cl}^{\prime}$ und des $\mathrm{ClO}_{3}{ }^{\prime}$ ausgeführt.

\section{Tabelle 2.}

Zersetzung einer $\mathrm{ClO}_{2}$-Lösung bei $100^{\circ} \mathrm{im}$ Dunkeln.

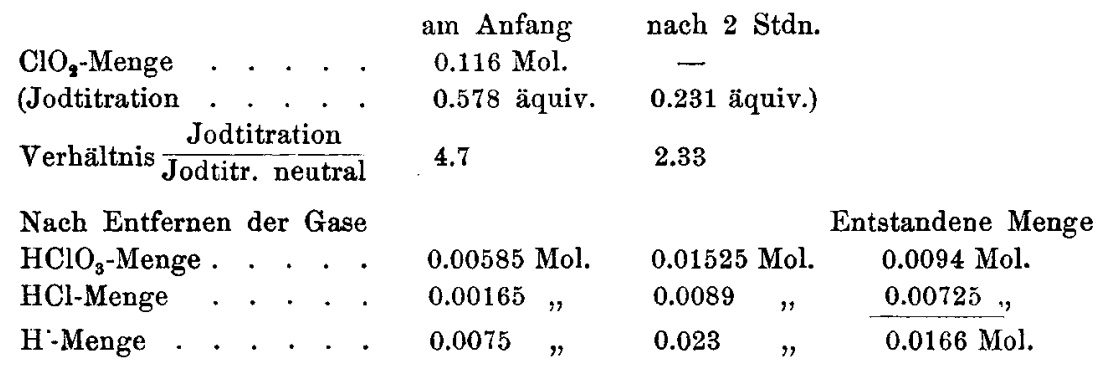


Aus diesen Zahlen lälst sich berechnen, dals etwa $60 \%$ des ursprünglichen $\mathrm{ClO}_{2}$ in Chlor umgesetzt worden ist; da $30 \%$ unzersetzt geblieben sind, folgt, dals etwa $85 \% \mathrm{ClO}_{2}$ in $\mathrm{Cl}_{2}$ und $\mathrm{O}_{2}$ zerfallen sind. Da das Verhältnis der entstandenen Säuren $\mathrm{HClO}_{3}$ nur 1.3 ist, während es bei der Zersetzung von $\mathrm{ClO}_{2} 5$ hätte sein müssen, so folgt daraus, dafs $\mathrm{Cl}_{2}$ mit zur Bildung der Säuren beizutragen hat.

Da ferner nur $8 \%$ des ursprünglichen $\mathrm{ClO}_{2}$ (soviel berechnet sich, wenn man das mitreagierende $\mathrm{Cl}_{2}$ berücksichtigt) in die Säuren sich umgesetzt haben, so ergibt sich daraus, dafs die Reaktion nur eine kleine Rolle spielt, möglicherweise nur als eine Folgereaktion.

Folgende kleine Tabelle gibt einen Überblick, welche $\mathrm{ClO}_{2}$ Mengen zur Bildung der gefundenen Reaktionsprodukte erforderlich sind:

\begin{tabular}{|c|c|c|}
\hline Gefu & ndene Menge & Entspr. $\mathrm{ClO}_{2}$-Menge \\
\hline $\mathrm{ClO}_{2}$ & $0.0356 \mathrm{Mol}$. & $0.0356 \mathrm{Mol}$. \\
\hline $1 / 2 \mathrm{Cl}_{2}$ & 0.053 & 0.053 \\
\hline Säure & 0.0166, & $0.0166, "$, \\
\hline
\end{tabular}
so dafs $0.011 \mathrm{ClO}_{2}$ durch Entweichen verloren gingen.

(S. Tabelle 3, S. 226.)

Aus diesen Resultaten folgt zunächst, dafs die Zersetzung von $\mathrm{ClO}_{2}$ in $\mathrm{Cl}_{2}$ und $\mathrm{O}_{2}$ die vorwiegende Reaktion ist; aber sie ist auch wahrscheinlich die primäre. Dafür spricht vor allem, dafs die sich bildenden Säuremengen mit der Zeit wachsen. Denn da das Verhältnis $\frac{\mathrm{HClO}_{3}}{\mathrm{HCl}}$ weder dem bei der Zersetzung des $\mathrm{ClO}_{2}$ allein, noch des $\mathrm{Cl}_{2}$ allein entspricht, lä sst sich das Anwachsen nur so erklären, dals das $\mathrm{ClO}_{2}$ mit dem $\mathrm{Cl}_{2}$ in einer Folgereaktion reagiert. Da $\mathrm{ClO}_{2}$ und $\mathrm{Cl}_{2}$ allein in Lösung relativ beständige Stoffe sind, beide zusammen aber nicht, so lälst sich vermuten, dafs eine Reaktion zwischen beiden vorhanden ist. Dies wurde schon oben erwähnt und im folgenden untersucht. Es soll nur noch auf die auffallende Tatsache aufmerksam gemacht werden, dafs das Verhältnis $\frac{\mathrm{HClO}_{3}}{\mathrm{HCl}}$ von einem 1 übersteigenden Wert beim Verlauf der Reaktion unter 1 sinkt. $\mathrm{Ob}$ die Zersetzung des $\mathrm{ClO}_{2}$ in $\mathrm{Cl}_{2}$ und $\mathrm{O}_{2}$ in der Gas- 
Tabelle 3.

Zersetzung einer $\mathrm{ClO}_{2}$-Lösung bei $100^{\circ}$ im Dunkeln.

Experimentelle Resultate Entstandene Mengen am Anfang Rohr 1, Rohr 2, Rohr 3, Rohr 4

\section{Zeit in Stunden}

$\mathrm{ClO}_{2}$-Menge

Jodtitration

Verhältnis

Jodtitration

0
0.127

0.637

\section{7}

0.0030

0.00185

0.00485
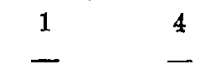

0.418

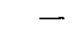

0.420

3.83

0.0058

0.0025

$0.0076^{1}$

\subsection{2}

0.0066

0.00388

0.0099

Berechnungen:

Verbältnis: $\frac{\text { entstandene } \mathrm{HClO}_{3}}{\text { entstandene } \mathrm{HCl}}$

Die gefund. $\mathrm{ClO}_{2}$-Menge entspr.

Die gefund. $\mathrm{Cl}_{2}$-Menge entspr.

Die gefund. Säuremenge entspr:

Gefunden anstatt $0.127 \mathrm{Mol}$.

2.32

1.70

0.77

0.76

0.078

0.078

0.001

0.030

0.032

0.030

$0.001 \mathrm{Mol} . \mathrm{ClO}_{2}$

0.008

0.116

$\begin{array}{lll}0.010 & 0.050 & 0.060\end{array}, ", \overline{\mathrm{ClO}}$

0.050

$\%$ des ursprüngl. $\mathrm{ClO}_{2}$, welches

$\left.\begin{array}{c}\text { mit Chlor reag. hat, um } \mathrm{HClO}_{3} \\ \text { und } \mathrm{HCl} \mathrm{zu} \mathrm{bilden}\end{array}\right\}$

$\%$ des ursprüngl. $\mathrm{ClO}_{2}$, welches

sich in $\mathrm{Cl}_{2}$ und $\mathrm{O}_{2}$ zersetzt hat etwa

34

$\%$ des verschwundenen $\mathrm{ClO}_{2}$,

welches sich in $\mathrm{Cl}_{2}$ und $\mathrm{O}$ zersetzt hat, etwa
5.2

5.7

32

80

$5.7 \quad 14.5 \quad 19.0 \%$

84

80

oder in der Flüssigkeitsphase sich vollzieht, war nicht zu entscheiden; wahrscheinlich tritt sie in beiden ein. Es mufs noch erwähnt werden, dafs der Vorgang unregelmäfsig ist und die Versuche nicht reproduzierbar sind.

\section{Die Reaktion $\mathrm{ClO}_{2}+1 / 2 \mathrm{Cl}_{2}+\mathrm{H}_{2} \mathrm{O}=\mathrm{HClO}_{3}+\mathrm{HCl}$.}

In folgenden Versuchen wurde die im Dunkeln verlaufende Zersetzung von Lösungen untersucht, welche $\mathrm{ClO}_{2}$ und Chlor in verschiedenen Mengen enthielten. Die anfängliche Zusammensetzung jeder Lösung wurde aus den abgemessenen Mengen der $\mathrm{ClO}_{2}$ und $\mathrm{Cl}_{2}$-Lösungen bestimmt und findet sich an der Spitze jeder Tabelle angegeben. Das Verhältnis zwischen den zwei Jodtitrationen wurde auch nach der zweiten im $\$$ I beschiebenen Methode (direkte Be-

'Die gefundenen „Säuremengen“ waren immer zu klein. (Siehe oben.) 
stimmung vcn $\mathrm{ClO}_{2}$ und $\mathrm{Cl}_{2}$ in der Ausgangslösung, Zugabe von Bikarbonat) :ur Kontrolle festgestellt, und zeigte genügende Übereinstimmung.

Die Versuche wurden in der üblichen Weise ausgeführt. Der Zweck der Messungen war den Quotient $\frac{\text { entstandene } \mathrm{HClO}_{3}}{\text { entstandene } \mathrm{HCl}} \mathrm{zu}$ bestimmen, welcher in der letzten Kolonne angegeben ist.

Das Verbältnis zwischen den reagierenden Substanzen, Chlordioxyd und Chlor wurde zwischen 3:1 und 1:3 variiert. Eine Gasbildung konnte in keinem Versuch konstatiert werden.

(S. Tabellen 4, 5 u. 6, S. 228 u. 229.)

Diese experimentellen Resultate erlauben folgende Schlüsse:

a) Es findet eine Reaktion zwischen $\mathrm{ClO}_{2}$ und $\mathrm{Cl}_{2}$ statt, bei der fast gleiche Mengen Chlorsäure und Salzsäure entstehen. So lange das Verhältnis zwischen der Chlordioxyd- und Chlorkonzentration sich zwischen $1: 3$ und $3: 1$ bewegt, ändert sich bei nicht allzu hoher Temperatur das Verhältnis $\frac{\mathrm{HClO}_{3}}{\mathrm{HCl}}$ nur von $0.9 \mathrm{zu} 1.1$. Es handelt sich also um die Reaktion:

$$
\mathrm{ClO}_{2}+{ }^{1 / 2} \mathrm{Cl}_{2}+\mathrm{H}_{2} \mathrm{O}=\mathrm{HClO}_{3}+\mathrm{HCl} \text {. }
$$

b) Eine Änderung der $\mathrm{ClO}_{2}$-Konzentration hat einen viel grölseren Einflufs auf die Geschwindigkeit der Reaktion als eine gleiche Änderung der Chlorkonzentration (vergl. Tabellen 5 u. 6).

c) Das Verhältnis $\frac{\mathrm{HClO}_{3}}{\mathrm{HCl}}$ ist am grörsten, wenn der $\mathrm{ClO}_{3}$ Überschuls am gröfsten ist, wie es auch in der Zersetzung des $\mathrm{ClO}_{2}$ bei $100^{\circ}$ gefunden wurde.

d) Mindestens zwei andere Reaktionen müssen angenommen werden:

1. Um die Entstehung eines $\mathrm{HClO}_{3}$. Überschusses in dem letzten Versuch, Tabelle 5, zu erklären, kann man annehmen, dafs die Reaktion:

$$
6 \mathrm{ClO}_{2}+3 \mathrm{H}_{2} \mathrm{O}=5 \mathrm{HClO}_{3}+\mathrm{HCl}
$$

durch das sich bildende $\mathrm{Cl}^{\prime}$ beschleunigt wird (siehe auch § IV).

2. Um die Entstehung eines HCl-Überschusses in Versuch 5, Tabelle 4 und in Tabelle $6 \mathrm{zu}$ erklären, läfst sich vielleicht die Reaktion: 
Tabelle 4.

Etwa äquivalente Mengen $\begin{array}{lll}\mathrm{ClO}_{2} & 0.50 \text { molar it.2 Liter } \\ \mathrm{Cl} & 0.44 \text { äquiv , " " }\end{array}$

\begin{tabular}{|c|c|c|c|c|c|c|c|c|c|}
\hline \multirow{2}{*}{ 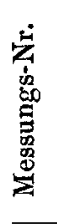 } & \multirow{2}{*}{ 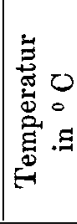 } & \multirow{2}{*}{ 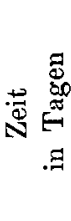 } & \multirow{2}{*}{ 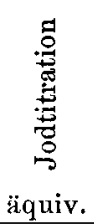 } & \multirow{2}{*}{ 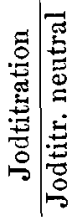 } & \multicolumn{4}{|c|}{ Konzentration in Millimolen } & \multirow{2}{*}{ 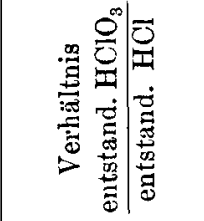 } \\
\hline & & & & & Säure & $\mathrm{Cl}^{\prime}$ & $\mathrm{ClO}_{\mathrm{s}}^{\prime}$ & $\begin{array}{c}\mathrm{ClO}_{3}^{\prime} \\
\text { berech. }\end{array}$ & \\
\hline 1 & 18 & 0 & $0.291^{1}$ & 3.2 & 5.47 & 1.72 & 3.7 & 3.75 & \\
\hline 2 & 18 & 8 & $0.26^{1}$ & 3.4 & 10.40 & 4.20 & 6.4 & 6.20 & $2.6 / 2.5=1.04$ \\
\hline 3 & 18 & 90 & $0.21^{\mathrm{I}}$ & - & 19.76 & 8.67 & - & 11.10 & $7.35 / 6.95=1.06$ \\
\hline 4 & 0 & 13 & 0.280 & 3.3 & 7.10 & 2.50 & 4.6 & 4.60 & $0.85 / 0.8=1.06$ \\
\hline 5 & $\left\{\begin{array}{r}0 \\
60\end{array}\right.$ & $\begin{array}{r}13 \\
1\end{array}$ & 0.226 & 3.4 & 29.9 & 16.4 & 13.4 & 13.5 & $8.9 / 13.9=0.67$ \\
\hline
\end{tabular}

Tabelle 5 .

$\begin{array}{llll}\mathrm{ClO}_{2} \text {-Überschüsse. } & 3 \mathrm{ClO}_{2}: 1 \mathrm{Cl} & \begin{array}{l}\text { Chlordioxyd } \\ \text { Chlor }\end{array} & 0.112 \text { molar. } \\ \text { Chlor } & 0.037 \text { äquiv. }\end{array}$

\begin{tabular}{|c|c|c|c|c|c|c|c|c|c|}
\hline \multirow{2}{*}{ 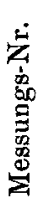 } & \multirow{2}{*}{ 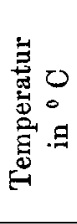 } & \multirow{2}{*}{ 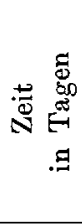 } & \multirow{2}{*}{ 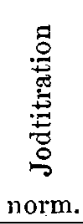 } & \multirow{2}{*}{ 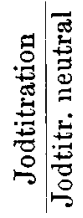 } & \multicolumn{4}{|c|}{ Konzentration in Millimolen } & \multirow{2}{*}{ 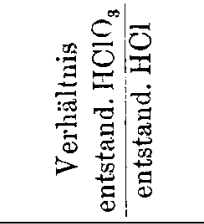 } \\
\hline & & & & & Säure & $\mathrm{Cl}^{\prime}$ & $\mathrm{ClO}_{3}^{\prime}$ & $\begin{array}{c}\mathrm{ClO}_{3}^{\prime} \\
\text { berech. }\end{array}$ & \\
\hline 1 & 18 & 0 & 0.597 & 4.0 & 11.0 & 3.57 & 7.5 & 7.4 & \\
\hline 2 & 18 & 4 & 0.572 & 一 & 19.7 & 7.7 & 12.1 & 12.0 & $4.6 / 4.1=1.12$ \\
\hline 3 & 18 & 39 & 0.401 & - & 60.2 & 26.7 & 34.1 & 33.5 & $26.6 / 23.1=1.15$ \\
\hline 4 & 18 & 111 & 0.250 & - & 104.7 & 38.0 & - & 66.7 & $59.3 / 34.4=1.72$ \\
\hline 5 & 18 & 112 & 0.250 & $4.8^{2}$ & 105.2 & 38.6 & 67.2 & 66.6 & $59.7 / 35.0=1.71$ \\
\hline 6 & 60 & 0.73 & 0.511 & - & 47.9 & 18.2 & 24.0 & 23.7 & $16.5 / 14.6=1.13$ \\
\hline 7 & 60 & 2.0 & 0.390 & - & 72.3 & 30.1 & 43.0 & 42.2 & $35.5 / 26.5=1.34$ \\
\hline
\end{tabular}

1 Die Lösung wurde in einer Flasche bei Zimmertemperatur aufbewahrt, und Proben 1, 2 und 3 zur Analyse genommen.

2 Das Verhältnis zwischen den zwei Jodtitrationen in Versuch 5, Tabelle 5 und Versuch 3, Tabelle 6 wurde nach der ersten Methode durch Analysieren des Gases bestimmt. Die Tatsache, dafs in Versuch 5, Tabelle 5, fast reines $\mathrm{ClO}_{2}$ vorhanden ist, zeigt, dafs die $\mathrm{ClO}_{2}$-Konzentration am Anfang gröfser als $0.112 \mathrm{Mol}$. und dafs die Chlorkonzentration kleiner als 0.037 normal war. 
Tabelle 6.

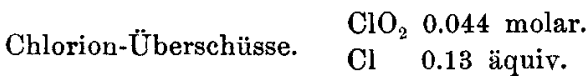

\begin{tabular}{|c|c|c|c|c|c|c|c|c|c|}
\hline \multirow{2}{*}{ 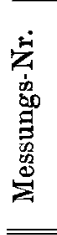 } & \multirow{2}{*}{ 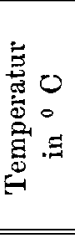 } & \multirow{2}{*}{ 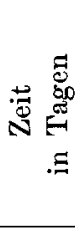 } & \multirow{2}{*}{ 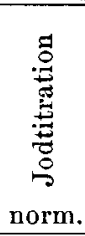 } & \multirow{2}{*}{ 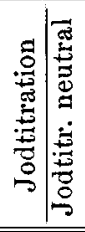 } & \multicolumn{4}{|c|}{ Konzentration in Millimolen } & \multirow{2}{*}{ 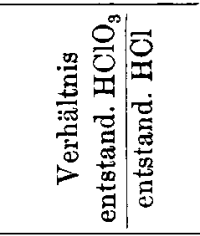 } \\
\hline & & & & & Säure & $\mathrm{Cl}^{\prime}$ & $\mathrm{ClO}_{3}^{\prime}$ & $\begin{array}{c}\mathrm{ClO}_{3}^{\prime} \\
\text { berech. }\end{array}$ & \\
\hline 1 & 18 & 0 & 51 & 2.1 & & & & & \\
\hline 2 & 18 & 46 & & - & & & 15.3 & 151 & $2.1 / 1$ \\
\hline 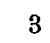 & 18 & 120 & 0.202 & $1.86^{1}$ & 57 & נס & - & 26.7 & $.5 /$ \\
\hline x & 60 & $\begin{array}{c}\text { Stdn } \\
19\end{array}$ & 0.284 & & 32.9 & 19.4 & 13.3 & 13.5 & 1 \\
\hline 5 & 60 & 51 & 0.233 & - & 58.0 & 34.2 & 23.0 & 23.8 & 0.64 \\
\hline
\end{tabular}

heranziehen.

$$
3 \mathrm{Cl}_{2}+3 \mathrm{H}_{2} \mathrm{O}=\mathrm{HClO}_{3}+5 \mathrm{HCl}
$$

e) Eine Temperaturerhöhung begünstigt die Salzsäurebildung. Tabellen 4 und 6, vergl. Versuch 3 mit Versuch 5. Tabelle 5, vergl. Versuch 5 mit 7.

Es ist merkwürdig, dafs die Zersetzung von $\mathrm{Cl}_{2}$ - und $\mathrm{ClO}_{2}$ Lösungen für sich allein sehr langsam verlaufende Vorgänge sind, während ein Zusatz von $\mathrm{ClO}_{2}$ die Zersetzung einer $\mathrm{Cl}_{2}$-Lösung beschleunigt und umgekehrt. Dies macht es sehr unwahrscheinlich, dafs es sich hier nur um diese zwei nebeneinander verlaufenden Vorgänge handelt, es spricht eher dafür, dafs hier eine stöchiometrische Reaktion in Frage kommt, zumal die Geschwindigkeit bei Gleichheit der $\mathrm{Cl}_{2}$ und $\mathrm{ClO}_{2}$-Menge ungefähr am grölsten ist und ferner das Verhältnis $\frac{\mathrm{HClO}_{3}}{\mathrm{HCl}}$ in einem weiten Bereich gleich 1 gefunden wurde. Am entschiedensten spricht aber für die Auffassung die Tatsache, dals die umgekehrte Realtion zwischen $\mathrm{HClO}_{3}$ und $\mathrm{HCl}$ bereits bekannt ist (siehe $\S \mathrm{V}$ ).

\section{Der Einflufs des Sonnenlichtes.}

Ich habe die Zersetzung im Sonnenlicht bisher nicht eingehend untersucht, möchte aber das anderweitig gewonnene Material im Anschlufs an meine übrigen Versuche mit ein paar Worten disku-

\footnotetext{
1 S. Note 2, S. 228.
} 
tieren. Es sei an dieser Stelle erwähnt, dafs ich, wegen der Lichtempfindlichkeit des $\mathrm{ClO}_{2}$, die auch in anderen Reaktionen zutage tritt, meine sämtlichen Versuche im Dunkeln ausgeführt habe.

Es ist bekannt, dafs Licht eine stark beschleunigende Wirkung auf die Zersetzung von $\mathrm{ClO}_{2}$-Gas und $\mathrm{ClO}_{2}$-Lösungen ausübt. POPPER ${ }^{1}$ fand, dals sie eine vollständige war und nimmt in offenen Gefälsen (wobei also das $\mathrm{Cl}_{2}$ entweichen kann) folgende Reaktion an:

$$
3 \mathrm{ClO}_{2}+\mathrm{H}_{2} \mathrm{O}=2 \mathrm{HClO}_{3}+1 / 2 \mathrm{O}_{2}+1 / 2 \mathrm{Cl}_{2} \text {. }
$$

Dafs hier wirklich die Chloratbildung mit dem Lichteinflufs etwas zu tun hat, ist unwahrscheinlich, und zwar aus folgenden Gründen:

1. Auch trockenes $\mathrm{ClO}_{2}$-Gas zerfällt in $\mathrm{Cl}_{2}$ und $\mathrm{O}_{2}$ im Sonnenlicht;

2. der Zerfall von $\mathrm{ClO}_{2}$ in $\mathrm{Cl}_{2}$ und $\mathrm{O}_{2}$ wurde bei höherer Temperatur von mir als der wahrscheinlich primäre Vorgang aufgefalst, und die Wirkung des Lichtes und der Temperaturerhöhung geht in vielen Erscheinungen parallel;

3. Gelegenheit für Chloratbildung ist vorhanden, wenn $\mathrm{ClO}_{2}$ und $\mathrm{Cl}_{2}$ nebeneinander vorhanden sind;

4. dals Popper in offenen Gefälsen nur wenig $\mathrm{Cl}^{\prime}$ fand, hat wohl seinen Grund darin, dals dieses mit dem jedenfalls vorhandenen $\mathrm{ClO}^{\prime}$ (dieses tritt, wie weiter unten ausgeführt ist, sehr wahrscheinlich auf, wenn $\mathrm{HClO}_{3}$ und $\mathrm{HCl}$ entstehen) $\mathrm{Cl}_{2}$ gibt, welches entweicht.

Demnach hat die reine Zersetzung in $\mathrm{Cl}_{2}$ und $\mathrm{O}_{2}$ das meiste für sich. Popper hat die gleiche Reaktion im Licht in geschlossenen Gefälsen untersucht und gelangt hier zu der komplizierten Gleichung:

$$
18 \mathrm{ClO}_{2}+9 \mathrm{H}_{2} \mathrm{O}=13 \mathrm{HClO}_{3}+3 \mathrm{O}_{2}+5 \mathrm{HCl} \text {. }
$$

Diese Gleichung ist als vollständiger Ausdruck der Tatsachen nicht nur an und für sich unwahrscheinlich, weil sie so kompliziert ist, und weil die Menge der Reaktionsprodukte nur am Ende und nicht während des Verlaufes bestimmt wurden (was allein über die wirklich verlaufenden Vorgänge $z u$ entscheiden erlaubt), sondern fordert auch im übrigen die gleiche Kritik heraus wie die oben genannte Gleichung.

1 Popper, Lieb. Ann. 227 (1884), 161; 231 (1885), 137. 
Erwähnt mag noch werden, dafs bei den PopPERschen Versuchen auch die Reaktion $\mathrm{Cl}_{2}+\mathrm{H}_{2} \mathrm{O}=2 \mathrm{HCl}+{ }^{1 / 2} \mathrm{O}_{2}$ eine Rolle spielen kann.

\section{Erörterung der möglichen Reaktionen.}

Folgende Reaktionen sind also bei der Zersetzung von Chlordioxyd in Betracht zu ziehen:

$$
\begin{aligned}
\text { I. } & \mathrm{ClO}_{2}=1 / 2 \mathrm{Cl}_{2}+\mathrm{O}_{2} \\
\text { II. } & 6 \mathrm{ClO}_{2}+3 \mathrm{H}_{2} \mathrm{O}=5 \mathrm{HClO}_{3}+\mathrm{HCl} \\
\text { III. } & \mathrm{ClO}_{2}+1 / 2 \mathrm{Cl}_{2}+\mathrm{H}_{2} \mathrm{O}=\mathrm{HClO} 3+\mathrm{HCl} \\
\text { IV. } & 3 \mathrm{Cl}_{2}+3 \mathrm{H}_{2} \mathrm{O}=\mathrm{HClO} 3+5 \mathrm{HCl} \\
\text { V. } & \mathrm{Cl}_{2}+\mathrm{H}_{2} \mathrm{O}=2 \mathrm{HCl}+1 / 2 \mathrm{O}_{2} .
\end{aligned}
$$

Von diesen sind die ersten drei isoliert und für sich allein untersucht worden. II wird durch Chlorion und durch platiniertes Platin beschleunigt; III ist die rascheste Reaktion, welche bei der Zersetzung einer $\mathrm{ClO}_{2}$-Chlorlösung im Dunkeln stattfindet. I findet bei höheren Temperaturen und wahrscheinlich im Sonnenlicht statt.

Reaktion IV wird wahrscheinlich durch platiniertes Platin beschleunigt. ${ }^{1}$ Es ist bekannt, dafs sie auch in Anwesenheit von Silberion stattfindet; nach Stas entsteht zugleich $\mathrm{AgClO}_{3}, \mathrm{AgCl}$ und $\mathrm{O}_{2}$, was ich bestätigen konnte.

Reaktion $V$ wird durch Licht beschleunigt, und ist aller Wahrscheinlichkeit nach eine Reaktion der unterchlorigen Säure $\mathrm{HClO}=$ $\mathrm{HCl}+{ }^{1} / 2 \mathrm{O}_{2} \cdot{ }^{2}$ Die drei Reaktionen II-IV sind wahrscheinlich nicht voneinander unabhängig. Es ist bekannt, dafs $\mathrm{Cl}_{2}$ in Gleichgewicht mit $\mathrm{HClO}$ steht; von II ist an anderer Stelle ${ }^{3}$ gezeigt worden, dals sie bei Gegenwart von $\mathrm{Cl}^{\prime}$ unter Bildung von $\mathrm{HClO}_{2}$ und $\mathrm{HClO}$ verläuft und von III werden die gleichen $Z$ wischenprodukte in $\S \mathrm{V}$ als wahrscheinlich in Betracht gezogen werden. Man wird also eine gegenseitige Beeinflussung der drei Reaktionen zu erwarten haben.

\section{§ III. Die Reaktion zwischen $\mathrm{ClO}_{2}$ und $\mathrm{OH}-\mathrm{Ion}$.}

Wie schon bekannt, können die Chloritlösungen durch die Reaktion zwischen $\mathrm{ClO}_{2}$ und $\mathrm{OH}^{\prime}$ hergestellt werden. Diese erfolgt nach der Gleichung:

1 SiR, Zeitschr. f. Elelktroehem. 11 (1905), 261.

2 Zeitschr. phys. Chem. 54 (1906), 463.

${ }^{3}$ Zeitschr. phys. Chen. $\mathbf{5 4}$ (1906) (unter Druck).

${ }^{4}$ Wie schon die Gleichung erkennen lälst, handelt es sich um eine Reaktion des $\mathrm{OH}^{\prime}$; deshalb verhalten sich allem Anschein nach $\mathrm{NH}_{3}$-Lösungen 


$$
2 \mathrm{ClO}_{2}+2 \mathrm{OH}^{\prime}=\mathrm{ClO}_{2}{ }^{\prime}+\mathrm{ClO}_{3}{ }^{\prime}+\mathrm{H}_{2} \mathrm{O} .
$$

Es wurde eine Reihe von Experimenten angestellt, um zunächst qualitativ den Einflufs der $\mathrm{OH}^{\prime}$-Konzentration festzustellen, die dann durch kinetische Versuche ergänzt wurden.

\section{Die Reaktion mit Natrium-Bikarbonat-Lösungen.}

Aus Versuchen von REYCHLER ${ }^{1}$ mit Kaliumbikarbonatlösungen ging schon hervor, dafs diese Reaktion nur sehr langsam rerlaufen würde. Ich habe dies durch folgenden Versuch bestätigt: etwa $4 \mathrm{~g}$ festes Natriumbikarbonat wurde zu einer 0.15 mol. $\mathrm{ClO}_{2}$-Lösung, welche etwas Schwefelsäure enthielt, gegeben. Das Rohr wurde zugeschmolzen und im Dunkeln bei $0^{0} 7$ Wochen lang gelassen. Eine merkliche Abnahme der Farbe liefs sich lange nicht konstatieren. Noch nach der genannten Zeit war die Lösung schwach gelb gefärbt. Beim Öffnen des Rohres wurde ein starker Überdruck $\left(\mathrm{CO}_{2}\right)$ beobachtet.

Die Analysen ergaben folgende Resultate (Tabelle 7). Alle Versuche sind auf Mol. pro Liter umgerechnet worden; nur die Oxydationswerte mufsten in Oxydations-(Normal-)Einheiten gegeben werden.

\section{Tabelle 7.}

Experimentelle Resultate

Oxydationswert der Lösung (Jodtitration) .

Nach Entfernen des Gases mit einem Luftstrom.
Am Anfang

$$
0.750
$$

$\begin{array}{lll}0.0 & 0.2672 \quad " \\ 0.0126 & 0.7135 \quad \text { " } \\ 0.0011 & 0.0033 \text { Mol. }\end{array}$

Am Ende 0.2762 norm. Oxydationswert (Jodtitration) . . . . . . Oxydationswert ( $\mathrm{FeSO}_{4}$-Titration) . . . . $\mathrm{AgNO}_{3}{ }^{\prime}$ Titration . . . . . . . . . .

Die Berechnungen ergeben:

Unzersetztes $\mathrm{ClO}_{2}$ nach der Reaktion. . . $0.0018 \mathrm{Mol}$.

Chlorat gebildet . . . . . . . . . . 0.0723 "

Chlorit gebildet. . . . . . . . . . 0.0668 ",

$\mathrm{Cl}^{\prime}$-Ion . . . . . . . . . . . . . . $\frac{0.0022,}{0.143 \mathrm{Mol}}$

Das gefundene "Gesamtchlor" entspricht also einer · $0.143 \mathrm{Mol}$. $\mathrm{ClO}_{2}$-Lösung Die ursprüngliche Lösung war. . . . . . . . 0.150 " „Überschufs“ an Chlorat über Chlorit . . . . . . 0.0055 "

$$
\text { Verhältnis } \frac{\text { Überschuls an Chlorat }}{\mathrm{Cl}^{\prime}-\text { Ion gebildet }}=\frac{0.0055}{0.0022}=2.5
$$

im Dunkeln wie solche von $\mathrm{NaOH}$ und $\mathrm{KOH}$; im Sonnenlicht tritt eine andere Reaktion auf: schneeweilse Nebel treten auf, die gelbe Farbe verschwindet viel rascher wie im Dunkeln; wieder ins Dunkle gebracht, lösen sich die Nebel schnell.

1 Zeitschr. phys. Chem. 54 (1906) (unter Druck). 
Diese Resultate zeigen, dafs nach 7 Wochen etwa $1.3 \%$ des Chlordioxyds noch unzersetzt geblieben war, und dafs mehr als $93 \%$ nach obiger Gleichung reagiert hatten, um gleiche Mengen von $\mathrm{ClO}_{3}{ }^{\prime}$ und $\mathrm{ClO}_{2}^{\prime}$ zu bilden.

Das übrige Dioxyd hat sich wahrscheinlich mit dem vorhandenen oder sich bildenden $\mathrm{Cl}_{2}$ (eine geringe Zersetzung nach der Gleichung $2 \mathrm{ClO}_{2}={ }^{1} / 2 \mathrm{Cl}_{2}+\mathrm{O}_{2}$ findet stets statt) unter $\mathrm{ClO}_{3}{ }^{\prime}$ und $\mathrm{Cl}^{\prime}$-Erzeugung umgesetzt.

Die Differenz zwischen der ursprünglichen $\mathrm{ClO}_{2}$-Konzentration und der berechneten kann dadurch erklärt werden, dafs etwas $\mathrm{ClO}_{2}$ beim Einfüllen des Rohres verloren ging, und dafs sich Natriumhypochlorit in kleinen Mengen gebildet hatte.

Der langsame Verlauf dieser Reaktion, welche mit Kalilauge in wenigen Stunden beendet wird, zeigt, dals die Geschwindigkeit von der $\mathrm{OH}^{\prime}$-Konzentration abhängig ist.

(Vergleiche die kinetischen Versuche unten.)

Es ist nicht ohne Bedeutung, dals ein Chlorit in einer Bikarbonat-Kohlensäurelösung beständig ist. Diese Tatsache machte es möglich, die Chloritstufe in den in der Einleitung erwähnten Reaktionen abzufangen. Im Vergleich mit den raschen Reaktionen, welche untersucht wurden, kam die langsame Reaktion zwischen $\mathrm{ClO}_{2}$ und Natriumbikarbonat nicht in Betracht.

Wie schon erwähnt, erleidet eine Lösung von chloriger Säure eine freiwillige Zersetzung und ist im allgemeinen eine sehr unbeständige Substanz. Eine $\mathrm{ClO}_{2}{ }^{\prime}$-Ionlösung bleibt dagegen monatelang unverändert. Folglich ist es sehr wahrscheinlich, dafs das Chlorit in der Bikarbonatlösung nur in der Form von $\mathrm{ClO}_{2}{ }^{\prime}$-Ionen vorhanden ist. Dies würde bedeuten, dafs chlorige Säure eine viel stärkere Säure ist als eine Bikarbonat-Kohlensäurelösung. Sie ist wahrscheinlich auch stärker als Kohlensäure selbst, da am Ende des Versuches viel $\mathrm{CO}_{2}$ und wenig $\mathrm{NaHCO}_{3}$ vorhanden war.

Dieser Schlufs wurde weiter noch bestätigt durch Versuche, in welchen Kohlensäure durch eine reine neutrale Chloritchloratlösung langsam geleitet wurde. Die mit $\mathrm{CO}_{2}$ gesättigte Lösung erlitt in drei Stunden praktisch keine Zersetzung, und es entstand kein $\mathrm{ClO}_{2}$. War aber unterchlorige Säure vorhanden, so wurden die Lösungen schnell gelb, da die oben erwähnte Reaktion nach der Gleichung:

$$
\mathrm{ClOH}+\mathrm{ClO}_{2} \mathrm{H}=\mathrm{ClO}_{2}+1 / 2 \mathrm{Cl}_{2}+\mathrm{H}_{2} \mathrm{O}
$$

vor sich geht. 


\section{Kinetische Untersuchung der Reaktion zwischen $\mathrm{ClO}_{2}$ und Natronlauge.}

Es war von Interesse, diese Reaktion zu verfolgen, weil möglicherweise ein hydrolytischer Vorgang der Form:

$$
2 \mathrm{ClO}_{2}+\mathrm{H}_{2} \mathrm{O}=\mathrm{HClO}_{2}+\mathrm{HClO}_{3}
$$

vorlag, der durch die Anwesenheit des $\mathrm{OH}-\mathrm{I}$ ons beeinflufst werden mochte.

Die Versuche wurden im Dunkeln bei $0^{\circ}$ angestellt und zwar mit sehr verdünnten Lösungen, um die Geschwindigkeit in mefsbare Gebiete zu bringen und allzu starke Wärmeentwickelungen zu vermeiden. Es wurden kleine Mengen von $\mathrm{ClO}_{2}$-Lösung ${ }^{1}$ mit überschüssiger, kohlensäurefreier $\mathrm{NaOH}$ (K $\mathrm{K}_{\triangle \mathrm{HLBADM}}$ ) vermischt, deren Gehalte nachträglich bestimmt wurden. Die anfängliche $\mathrm{ClO}_{2}$ Konzentration ergab sich aus der $\mathrm{ClO}_{2}{ }^{\prime}$-Konzentration nach Beendigung der Reaktion und zwar war sie ${ }^{10} / 4$ der letzteren, in Oxydationseinheiten gerechnet (vergl. REYchler). Diese und die in gewöhnlicher Weise bestimmte $\mathrm{OH}^{\prime}$-Konzentration finden sich an der Spitze der Tabelle. Es wurde in $300 \mathrm{ccm}$ fassende Glasflaschen (mit Stöpseln) gearbeitet und die zu analysierende Flüssigkeitsmenge mit einer grofsen, rasch auslaufenden Pipette (meist $20 \mathrm{ccm}$ ) zu einer sauren JK-Lösung gegeben; das ausgeschiedene Jod wurde mit sehr verdünntem (0.01) Thiosulfat titriert; die Konzentration wird in den Tabellen in Mol. pro Liter angefübrt.

Es sind nicht alle Versuche angeführt, sondern nur zwei typische ausführlich; für die anderen sind blofs die Anfangskonzentrationen und die Konstante gegeben. Wo $\mathrm{OH}^{\prime}$-Ion im Überschurs war, erwiesen sich die Versuchsergebnisse als reproduzierbar.

Es zeigte sich, dals der Vorgang nach der Gleichung:

$$
\frac{d x}{d t}=-\frac{d\left(\mathrm{ClO}_{2}\right)}{d t}={ }_{3} k\left(\mathrm{ClO}_{2}\right)^{2}\left(\mathrm{OH}^{\prime}\right)
$$

verlief; wurde $\mathrm{OH}^{\prime}$ konstant im Überschufs gehalten, so gilt $\frac{d x}{d t}=$ ${ }_{2} k(A-x)^{2}$, wo $A$ die Anfangskonzentration des $\mathrm{ClO}_{2}$ und ${ }_{2} k={ }_{3} k(B-x)$, wo $B$ die Anfangskonzentration des $\mathrm{OH}^{\prime}$ bedeutet.

1 Es wurden sowohl reine $\mathrm{CO}_{2}$-haltige Lösungen, wie die nach der Methode von REYCHLER hergestellten $\mathrm{Cl}_{2}$-haltigen verwendet. 
Die integrierte Gleichung ist:

$$
2^{k}=\frac{1}{t_{2}-t_{1}} \frac{\left(x_{2}-x_{1}\right)}{\left(A-x_{1}\right)\left(A-x_{2}\right)}
$$

wo die Indizes 1 und 2 auf je zwei avfeinander folgende Beobachtungen weisen, die miteinander kombiniert werden. Um zu zeigen, dals die Gleichung:

$$
\frac{d x}{d t} 2.3={ }_{1} k(A-x), \text { integriert }{ }_{1} k=\frac{1}{t_{2}-t_{1}} \log \frac{A-x_{1}}{A-x_{2}}
$$

nicht gilt, sind die ${ }_{1} k$-Werte mit angegeben.

Tabelle 8 .

\begin{tabular}{rcccc}
\multicolumn{2}{c}{ (Versuch 2, } & \multicolumn{3}{c}{$A=0.00360$ Mol. } \\
Tabelle 10) & & $B=0.0596 \quad "$ \\
$t$ & $(A-x) \cdot 10^{3}$ & ${ }_{1} k \cdot 10^{3}$ & ${ }_{2} k$ & ${ }_{3} k=\frac{{ }_{2} k}{B-x}$ \\
0 & $(3.60)$ & & & \\
1 & 3.10 & & & \\
6 & 2.43 & 21.0 & 17.8 & 305 \\
12 & 1.91 & 17.5 & 18.6 & 321 \\
20 & 1.47 & 14.1 & 19.1 & 333 \\
36 & 0.96 & 11.5 & 22.6 & 396 \\
50 & 0.73 & 8.3 & 23.0 & 405 \\
80 & 0.43 & 7.6 & 32.2 & 589
\end{tabular}

Für die erste Hälfte der Reaktion ${ }_{2} k=18.5{ }_{3} k=320$.

Tabelle 9.

\begin{tabular}{rcccc}
\multicolumn{2}{c}{$\begin{array}{c}\text { Versuch 7, } \\
\text { Tabelle 11) }\end{array}$} & \multicolumn{3}{c}{$\begin{array}{c}A=0.00304 \\
B=0.0610 \quad \text { Mol. (R) }\end{array}$} \\
$t$ & $(A-x) \cdot 10^{3}$ & ${ }_{1} k \cdot 10^{3}$ & ${ }_{2} k$ & ${ }_{3} k=\frac{{ }_{2} k}{B-x}$ \\
0 & $(3.04)$ & & & \\
1 & 2.52 & & & \\
5 & 2.14 & 17.7 & 17.6 & 293 \\
10 & 1.80 & 15.2 & 17.7 & 296 \\
15 & 1.56 & 12.3 & 17.45 & 292 \\
25 & 1.187 & 11.8 & 18.45 & 311 \\
52 & 0.692 & 8.7 & 20.5 & 350 \\
100 & 0.367 & 5.7 & 23.6 & 404
\end{tabular}

Für die erste Hälfte der Reaktion ist ${ }_{2} k=17.8,{ }_{3} k=295$. 
Es ist klar, dafs die Reaktion in erster Annäherung von der zweiten Ordnung in bezug auf Chlordioxyd ist. Der Gang des ${ }_{2} k$ wird erheblich, erst nachdem sich 70 bis $80 \%$ des Chlordioxyds umgewandelt haben, ${ }_{1} k$ dagegen zeigt einen Gang von Anfang an.

Folgende Tabellen geben die Werte der ,Konstanten' ${ }_{1} k \times 10^{3}$, ${ }_{2}^{k}$ und ihren Gang für verschiedene Versuchsreihen. Werte nach einer Umwandlung von $80 \%$ sind nicht berücksichtigt. Die Mittelwerte von ${ }_{2} k$ und ${ }_{3} k$ (in den letzten zwei Spalten) sind für die erste Hälfte der Reaktion berechnet. Die folgenden Tabellen lassen ferner noch den Einflufs von Chlor-, Chlorion-, Chlorationzusätzen und der Temperatur erkennen.

Tabelle 10.

Versuche mit reinen Chlordioxydlösungen.

\begin{tabular}{ccccccc} 
Vers.-Nr. & $A \cdot 10^{3}$ & $B \cdot 10^{3}$ & ${ }_{1} k \cdot 10^{3}$ & ${ }_{2} k$ & \multicolumn{2}{c}{ Mittelwerte } \\
1 & 1.70 & 20.1 & $35-24$ & 76 (steigt) & 76 & ${ }_{3} k$ \\
2 & 3.60 & 59.8 & $21-8$ & $18-23$ & 18.5 & 380 \\
3 & 1.86 & 38.0 & $8-4$ & $12-16$ & 14 & 370 \\
4 & 5.50 & 17.0 & $24-3$ & $4.9-3.8$ & 4.8 & 310
\end{tabular}

Tabelle 11.

Versuche mit chlorhaltigen Lösungen $(R)$.

\begin{tabular}{|c|c|c|c|c|c|}
\hline Vers.-Nr. & $A \cdot 10^{3}$ & $B \cdot 10^{8}$ & ${ }_{1} k \cdot 10^{3}$ & ${ }_{2} k$ & ${ }_{2} k$ \\
\hline 5 & 2.80 & 202 & $42-22$ & $63-83$ & 63 \\
\hline 6 & 1.87 & 62 & & $19-23$ & 21 \\
\hline 7 & 3.04 & 61 & $17-6$ & $17.6-22$ & 17.6 \\
\hline 8 & 3.20 & 60 & $17-6$ & $16-18$ & 17 \\
\hline 9 & 2.55 & 36 & $10-5$ & $10-12$ & 10.4 \\
\hline 10 & 2.50 & 18 & $5-2$ & 5.6 & 5.6 \\
\hline
\end{tabular}

Tabelle 12.

Einflufs von Chlor; zugegebenes Chlor 0.00006 norm.

$\begin{array}{clcccccc}\text { Vers.-Nr. } & A \cdot 10^{3} & B \cdot 10^{3} & { }_{1} k \cdot 10 & { }_{2} k & { }_{2} k & { }_{3}^{k} & \text { Vergleiche } \\ 11 & 3.46 & 60 & 21-7 & 17-23 & 18.8 & 320 & \text { Nr. } 2 \\ 12 & 3.02(\mathrm{R}) & 60 & 16-5 & 17-20 & 17.5 & 300 & n 7\end{array}$

Tabelle 13.

Einflufs von $\mathrm{Cl}^{\prime}$ und $\mathrm{ClO}_{\mathbf{3}}$ '.

Versuch 13. NaCl $1.0 \mathrm{Mol}$.

"14. $\mathrm{NaClO}_{3} \quad 0.5$,

Vers.-Nr. $\quad A \cdot 10^{3} \quad B \cdot 10^{3} \quad{ }_{1} k \cdot 10_{3} \quad{ }_{2} k \quad{ }_{2} k \quad{ }_{3}^{k} \quad$ Vergleiche

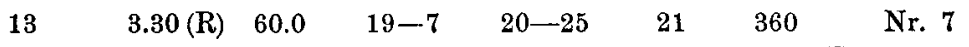

$14 \quad 3.10(\mathrm{R}) \quad 58.6 \quad 21-9 \quad 25-33 \quad 26 \quad 450$ (Titration nicht mehr sehr genau) 
Tabelle 14.

Einflufs der Temperatur.

Temperatur $=19^{\circ}$.

\begin{tabular}{|c|c|c|c|c|c|c|}
\hline Vers.-Nr. & $A \cdot 10^{3}$ & $B \cdot 10^{3}$ & ${ }_{1} k \cdot 10$ & ${ }_{2} k$ & ${ }_{2} k$ & ${ }_{3} k$ \\
\hline 15 & $3.12(\mathrm{R})$ & 60.6 & $48-18$ & $51-60$ & 54 & 920 \\
\hline 16 & $3.13(\mathrm{R})$ & 58.6 & $46-22$ & $52-80$ & 56.0 & 980 \\
\hline
\end{tabular}

Berechneter Temperaturkoeffizient $\frac{{ }_{3} k T+10}{{ }_{3} k T}=$ (ungefähr) 1.81 .

Es folgt aus diesen Resultaten, dafs die Geschwindigkeit der Reaktion in Anwesenheit überschüssigen Alkalis streng proportional der ersten Potenz der $\mathrm{OH}^{\prime}$-Konzentration und annähernd proportional der zweiten Potenz der $\mathrm{ClO}_{2}$-Konzentration ist. Die Anwesenheit kleiner Hypochloritmengen hat keinen Einflufs auf den Verlauf der Reaktion. Da die sehr konzentrierten $\mathrm{Cl}^{\prime}$ - und $\mathrm{ClO}_{3}{ }^{\prime}$-Lösungen nur eine kleine beschleunigende Wirkung zeigen, können diese Ionen in verdünnter Lösung nur einen verschwindend kleinen Einflufs haben. Der Temperaturkoeffizient für $10^{\circ}$ ist etwas gröfser als 1.8.

Die Zunahme der Konstanten ${ }_{2} k$ und ${ }_{3} k$ während eines Versuches, und besonders die starke Zunahme in den letzten Messungen bedeutet, dafs in einer sehr verdünnten $\mathrm{ClO}_{2}$-Lösung die Reaktion eine gröfsere Geschwindigkeit hat, als der Gleichung:

$$
\frac{d x}{d t}={ }_{3} K(A-x)^{2}(B-x) \text { entspricht. }
$$

In der Tat zeigen die Resultate, dals die Konstanten $\left({ }_{3} k\right)$ in den verschiedenen Versuchen zunehmen, je stärker die Anfangskonzentrationen $(A)$ abnehmen. Es wurde versucht, diesen Einflufs zu verfolgen und zu dem Zweck Versuche mit kleinen annähernd äquivalenten Mengen von $\mathrm{ClO}_{2}$ und $\mathrm{OH}^{\prime}$ angestellt; hier mufste die Reaktionsordnung niedriger als 3 und die Konstante höher als 300 gefunden werden. Dies war der Fall (zwei Versuche ergaben etwa 600 ), aber die Versuche liefsen sich so schlecht reproduzieren, dals auf sie nicht näher eingegangen werden soll.

Schliefslich mag kurz der wahrscheinliche Mechanismus dieser Reaktion besprochen werden; primär dürfte wohl der Vorgang $2 \mathrm{ClO}_{2}+\mathrm{OH}^{\prime}=\mathrm{ClO}_{3}{ }^{\prime}+\mathrm{HClO}_{2}$ statthaben, an den sich die rasche Folgereaktion $\mathrm{HClO}_{2}+\mathrm{OH}^{\prime}=\mathrm{ClO}_{2}{ }^{\prime}+\mathrm{H}_{2} \mathrm{O}$ anschlielst; die Bruttogleichung ist dans:

$$
2 \mathrm{ClO}_{2}+2 \mathrm{OH}^{\prime}=\mathrm{ClO}_{3}^{\prime}+\mathrm{ClO}_{2}^{\prime}+\mathrm{H}_{2} \mathrm{O} .
$$

Z. anorg. Chem. Bd. 48 . 
Die Mitwirkung des $\mathrm{OH}^{\prime}$ ist kein Beweis gegen, aber auch kein Beweis für die Existenz des hydrolytischen Gleichgewichtes:

$$
2 \mathrm{ClO}_{2}+\mathrm{H}_{2} \mathrm{O}=\mathrm{HClO}_{2}+\mathrm{HClO}_{3} \text {. }
$$

\section{\& IV. Die Zersetzung einer sauren Chlorat-Chloritlösung.}

1. Gründe für die Existenz des Gleichgewichtes $2 \mathrm{ClO}_{2}+\mathrm{H}_{2} \mathrm{O}=$

$$
\mathrm{HClO}_{3}+\mathrm{HClO}_{2} \text {. }
$$

Die Zersetzung einer angesäuerten Chlorat-Chloritlösung wurde untersucht, um einen positiven Beweis für die Existenz des Gleichgewichtes:

zu erbringen.

$$
2 \mathrm{ClO}_{2}+\mathrm{H}_{2} \mathrm{O}=\mathrm{HClO}_{3}+\mathrm{HClO}_{2}
$$

Bis jetzt ist dies Gleichgewicht aus folgenden Gründen als etwas Selbstverständliches betrachtet worden:

a) Gleiche Mengen von Chlorit und Chlorat entstehen in der Reaktion zwischen $\mathrm{ClO}_{2}$ und $\mathrm{OH}^{\prime}$-Ion und beim Ansäuern einer Chlorat-Chloritlösung wird die Flüssigkeit gelb: ein Zeichen, dafs $\mathrm{ClO}_{2}$ jedenfalls vorhanden ist.

b) Die Analogie mit Stickstoffperoxyd. ${ }^{1}$

$\mathrm{Zu}$ diesen können als Resultate meiner Untersuchungen folgende hinzugefügt werden.

c) Die Geschwindigkeit der Bildung von $\mathrm{ClO}_{2}$ in einer sauren Chlorit-Chloratlösung wird durch Zusatz von Kaliumchlorat oder von Schwefelsäure beschleunigt und ist gröfser in einer konzentrierten Chloritlösung, als in einer verdünnten. Auf Grund der Untersuchung des Verlaufes der Reaktion zwischen $\mathrm{ClO}_{2}$ und $\mathrm{OH}^{\prime}$ (§ III) ist folgende kinetische Gleichung zu erwarten:

$$
\frac{d\left(\mathrm{ClO}_{2}\right)}{d t}=K\left(\mathrm{ClO}_{3}{ }^{\prime}\right)\left(\mathrm{ClO}_{2}{ }^{\prime}\right)\left(\mathrm{H}^{*}\right),
$$

mit der der obenerwähnte Einflufs von $\mathrm{ClO}_{3}{ }^{\prime}, \mathrm{H}^{\cdot}$ und $\mathrm{ClO}_{2}{ }^{\prime}$ qualitativ übereinstimmt.

d) Der anderen Ortes ${ }^{2}$ besprochene Umstand, daf's $\mathrm{ClO}_{2}$ sich $\operatorname{dem} \mathrm{Cl}_{2}$ auffallend analog verhält.

${ }^{1}$ Siehe Gilberts Ann. 52 (1816), 197. Nachschrift.

2 Zeitschr. phys. Chem. 54 (1906) (unter Druck). 
Es mag noch erwähnt werden, dafs OEcHsLI ${ }^{1}$ zur Erklärung der Bildung von $\mathrm{ClO}_{2}$ aus $\mathrm{ClO}_{3} \mathrm{H}$ und konzentrierter Schwefelsäure eine primäre Bildung von chloriger Säure mit der Folgereaktion:

angenommen hat.

$$
\mathrm{ClO}_{3} \mathrm{H}+\mathrm{ClO}_{2} \mathrm{H}=2 \mathrm{ClO}_{2}+\mathrm{H}_{2} \mathrm{O}
$$

\section{Gegengründe.}

Das nähere Eingehen auf diese Beweisgründe und die Untersuchung der Zersetzung von Chlorit-Chloratlösungen lehrten aber, dafs, mit der möglichen Ausnahme (c), keiner dieser Beweisgründe Stich hält.

Es finden andere Reaktionen statt, nicht blofs $\mathrm{ClO}_{2}-\mathrm{Bildung}$. Chlor findet sich stets als Reaktionsprodukt, und die Chlorsäurekonzentration nimmt immer zu, anstatt ab. Mit anderen Worten scheint sich in einer wässerigen Lösung nur die chlorige Säure zu zersetzen, und die Entstehung von $\mathrm{ClO}_{2}$ (a) ist deshalb kein Beweis, dals eine Reaktion zwischen Chlorsäure und chloriger Säure stattfindet.

Geht sie überhaupt vor sich, so kann sie nur eine sehr geringe Geschwindigkeit besitzen, da in einer verdünnten Chlorigsäurelösung in Anwesenheit von Chlorsäure, praktisch kein $\mathrm{ClO}_{2}$ in einer Stunde entstand. Die geringe Geschwindigkeit lälst sich nicht daraus erklären, dals man in der Nähe des Gleichgewichtes sich befindet, denn allmählich wurden trotzdem grolse Mengen von $\mathrm{ClO}_{2}$ gebildet. Was Punkt (b) betrifft, so zeigt schon die Molekulargewichtsbestimmung. dals man die Analogie mit $\mathrm{N}_{2} \mathrm{O}_{4}$ nicht sehr weit treiben darf. Die zweifellos bestehende Analogie mit $\mathrm{Cl}_{2}$ hält hier auch nicht Stich: denn gerade das für $\mathrm{Cl}_{2}$ so charakteristische hydrolytische Gleichgewicht:

$$
\mathrm{Cl}_{2}+\mathrm{H}_{2} \mathrm{O}=\mathrm{HClO}+\mathrm{HCl}
$$

spielt bei $\mathrm{ClO}_{2}$ in der analogen Form:

$$
2 \mathrm{ClO}_{2}+\mathrm{H}_{2} \mathrm{O}=\mathrm{HClO}_{2}+\mathrm{HClO}_{3}
$$

nicht die gleiche Rolle.

Positive Gründe gegen seine Existenz fanden sich nicht vor, wohl aber müssen die betreffenden Reaktionen im Vergleich zu anderen vorkommenden sehr langsam verlaufen.

1 Zeitschr. f. Elektrochem. 9 (1903), 807. 


\section{Reaktionen zwischen $\mathrm{HClO}_{3}$ and $\mathrm{HClO}_{2}$.}

Es wurde schon erwähnt, dafs in Lösungen von $\mathrm{HClO}_{3}$ und $\mathrm{HClO}_{2}$ beim Ansäuern mit $\mathrm{H}_{2} \mathrm{SO}_{4} \mathrm{ClO}_{2}$-Bildung langsam erfolgt und durch $\mathrm{Cl}^{\prime}, \mathrm{ClO}^{\prime}, \mathrm{ClO}_{3}{ }^{\prime}, \mathrm{H} \cdot$ und wahrscheinlich $\mathrm{ClO}_{2}{ }^{\prime}$ und ferner durch Temperaturerhöhung beschleunigt wird. Wie kompliziert die Zersetzung ist, ersieht man aus der ebenfalls schon genannten Tatsache, dafs der Oxydationswert JK gegenüber ständig abnimmt, so dafs eine $\mathrm{ClO}_{3} \mathrm{H}-\mathrm{Bildung}$ angenommen werden muls, und dafs gleichzeitig $\mathrm{Cl}_{2}$ und $\mathrm{Cl}^{\prime}$ auftreten.

Der Zweck der nachfolgenden Versuche war, die verschiedenen Reaktionsprodukte ihrer Menge nach festzustellen, um daraus auf die wahrscheinlich stattfindenden Reaktionen schliefsen zu können.

Es wurden $10 \mathrm{ccm}$ einer $0.082 \mathrm{~mol} . \mathrm{ClO}_{2}{ }^{\prime}$-Lösung, die die gleiche Chloratmenge enthielt und $0.024 \mathrm{n}$. bezüglich des $\mathrm{OH}^{\prime}$ war, mit $10 \mathrm{ccm}$ $\mathrm{H}_{2} \mathrm{O}$ bezw. $\mathrm{NaClO}_{3}$, bezw. NaCl-Lösung und dann mit einer bekannten Menge (meistens $1 \mathrm{ccm}$ ) einer doppeltnormalen $\mathrm{H}_{2} \mathrm{SO}_{4}$-Lösung rermischt. Die Lösungen wurden hierdurch sauer und der Säureüberschufs findet sich in der zweiten Spalte der Tabellen.

$\mathrm{Da}$ es notwendig war, die Gesamtmenge der entstehenden Reaktionsprodukte kennen zu lernen, wurden zur Analyse alle Gase $\left(\mathrm{ClO}_{2}\right.$ und $\left.\mathrm{Cl}_{2}\right)$ aus den Lösungen durch einen ziemlich raschen Luftstrom entfernt und ohne Verlust in eine neutrale KJ-Lösung geleitet (vergl. \& I). Das Verhältnis der Jodtitrationen gibt wie immer die relative Menge $1 / 2 \mathrm{Cl}_{2}$ auf $\mathrm{ClO}_{2}$ (Spalte 9 der Tabellen) und daraus folgt die $\mathrm{ClO}_{2} \mathrm{H}$-Menge, die unter Bildung dieser beiden Gase sich zersetzt hat (dies ist „Jodtitration“, Spalte 6). Die unzersetzt gebliebene $\mathrm{HClO}_{2}$-Menge wurde durch eine Jodtitration der entgasten Lösung festgestellt (Spalte 4) und aus diesen und dem ursprünglichen Oxydationswert folgt die $\mathrm{HClO}_{3}$-Menge (Spalte 5). Alle Resultate sind in ccm 0.1 norm. Thiosulfatlösung ausgedrückt, deren Summe (Spalte 4, 5 und 6) den ursprünglichen Oxydationswert ergeben mufs, nämlich $32.8 \mathrm{ccm}$ Thiosulfat.

\section{(S. Tabelle 15, S. 241.)}

Die quantitativen Versuche bestätigen zunächst das qualitative Ergebnis bezüglich der Entstehung von $\mathrm{ClO}_{2}, \mathrm{Cl}_{2}, \mathrm{HClO}_{3}$ und $\mathrm{Cl}^{\prime}$.

Alle Reaktionen, die man zur Erklärung der $\mathrm{Cl}_{2}$-Bildung heranziehen kann, liefern zu viel $\mathrm{Cl}_{2}$ : da es ausgeschlossen erscheint, dafs $\mathrm{Cl}_{2}$ in einer Folgereaktion verschwindet (die Reaktion mit $\mathrm{ClO}_{2}$ 
Tabelle 15.

Die Zersetzung einer $\mathrm{HClO}_{2}-\mathrm{HClO}_{3}$-Lösung im Dunkeln.

Oxydationswert des $\mathrm{ClO}_{2}{ }^{\circ}=32.8 \mathrm{~cm} \mathbf{n} / 10$ Thiosulfat.

$\begin{array}{rrrrrrrrr}1 & 2 & 3 & 4 & 5 & 6 & 7 & 8 & 9\end{array}$

Ver- Säurekonz. Zeit Unzers. Gebild. Jod- Jod- Ver- Clsuchs- am Anfang in chlorig. Chlors. titra- titration hält- Gehalt auf

$\mathrm{Nr}$. (etwa) Minut. Säure (ber.) tion neutr. nis $100 \mathrm{ClO}_{2}$

\begin{tabular}{llrlrrrrl}
1 & $0.04 \mathrm{n}$. & 30 & 20.4 & 4.2 & 8.20 & 1.92 & 4.27 & $16 \%$ \\
2 & 0.08 & 60 & 10.9 & 8.1 & 13.75 & 3.10 & 4.43 & 11 \\
3 & 0.08 & 70 & 20.0 & 4.8 & 7.86 & 1.81 & 4.34 & 13.5 \\
4 & 0.08 & 150 & 14.3 & 6.7 & 11.75 & 2.70 & 4.35 & 13.5 \\
\hline
\end{tabular}

$\mathrm{NaClO}_{\mathrm{B}}$-Konzentration, 2.4 Mol. in der Lösung.

\begin{tabular}{rrrrrrrrc}
5 & 0.09 & 60 & 11.5 & 7.3 & 13.95 & 3.10 & 4.50 & 9 \\
6 & 0.40 & 90 & 0.0 & 4.4 & 28.35 & 6.75 & 4.20 & 19 \\
\hline etwa 14
\end{tabular}

NaCl-Konzentration, 2.4 Mol. in der Lösung.

\begin{tabular}{ccccccccc}
7 & 0.09 & - & - & - & 10.95 & 2.47 & 4.43 & 11 \\
8 & 0.09 & 60 & 0.1 & 2.1 & 30.60 & 6.82 & 4.49 & 9 \\
9 & 0.18 & 45 & 0.4 & 2.5 & 29.50 & 6.40 & 4.61 & 6 \\
\hline
\end{tabular}

ist zu langsam), bleibt nur übrig anzunehmen, dals neben den chlorliefernden Reaktionen noch solche verlaufen, die nur $\mathrm{ClO}_{2}$ geben. Zur Erklärung genügt also der Vorgang:

$$
4 \mathrm{ClO}_{2} \mathrm{H}=3 \mathrm{ClO}_{2}+1 / 2 \mathrm{Cl}_{2}+2 \mathrm{H}_{2} \mathrm{O}
$$

nicht, da er etwa $33 \% \mathrm{Cl}_{2}$ entstehen läfst, statt der $6-16 \%$, die beobachtet wurden. Es könnte schliefslich jede chlorliefernde Reaktion als Nebenreaktion stattfinden und zwar in um so geringerem Betrage, je mehr Chlor sie liefert, so dafs als einzige Forderung bestehen bleibt, dafs jedenfalls eine Hauptzersetzung $\mathrm{ClO}_{2}$ entstehen lälst, dals also auch die Reaktion: ${ }^{1}$

$$
\mathrm{H} \cdot+\mathrm{ClO}_{3}{ }^{\prime}+\mathrm{HClO}_{2}=2 \mathrm{ClO}_{2}+\mathrm{H}_{2} \mathrm{O}
$$

von statten geht. Die Chloratbildung wird verständlich bei Annahme einer Reaktion:

$$
3 \mathrm{HClO}_{2}=2 \mathrm{HClO}_{3}+\mathrm{HCl} \text {. }
$$

1 Möglicherweise spielt noch die Reaktion:

hierbei eine Rolle.

$$
5 \mathrm{ClO}_{2} \mathrm{H}=4 \mathrm{ClO}_{2}+\mathrm{HCl}+2 \mathrm{H}_{2} \mathrm{O}
$$


Was die beschleunigenden Einflüsse verschiedener Stoffe betrifft, so wird die Wirkung von $\mathrm{ClO}_{3}{ }^{\prime}, \mathrm{ClO}_{2}{ }^{\prime}$ und $\mathrm{H} \cdot$ durch Gleichung 2 einleuchtend. Nicht zu erklären auf Grund der bisher bekannten Tatsachen ist die Beschleunigung der $\mathrm{ClO}_{2}$-Bildung durch $\mathrm{Cl}^{\prime}$.

Weitere Aufklärung darf man nach diesen Vorversuchen von einer Untersuchung der Zersetzung reiner $\mathrm{ClO}_{2} \mathrm{H}$-Lösungen erhoffen, vor allem, wenn man die Reaktionen von reinem $\mathrm{KClO}_{2}$ verfolgt; auch die Untersuchung der Reaktion zwischen $\mathrm{HClO}_{3}$ und Oxalsäure bezw. Schwefelsäure dürfte weiter führen.

\section{§ V. Die Reaktion zwischen Chlorsäure und Salzsäure.}

\section{Versuche Schacherls.}

Bekanntlich entsteht in der Reaktion $z$ wischen Chlorsäure und verdünnter Salzsäure ein Gemisch von Chlordioxyd und Chlor. SchacherL ${ }^{1}$ liels Schwefelsäure auf Kaliumchlorat und Natrium- resp. Kaliumchlorid, einwirken und fand, als er die Chlormetallmenge verminderte, dafs der Chlordioxydgehalt des Gases sich allmählich vermehrte und dem Grenzfall von $2 \mathrm{Vol} . \mathrm{ClO}_{2}: 1 \mathrm{Vol} . \mathrm{Cl}_{2}$ sich näherte.

Tabelle 16.

\section{Salzgemisch.}

$1 \mathrm{Mol} . \mathrm{KClO}_{3}: 5 \mathrm{Mol} . \mathrm{NaCl}$

$1 \mathrm{Mol} . \mathrm{KClO}_{3}: 1 \mathrm{Mol} . \mathrm{NaCl}$

2 Mol. $\mathrm{KClO}_{3}: 1 \mathrm{Mol}$. NaCl

$4 \mathrm{Mol} . \mathrm{KClO}_{3}: 1 \mathrm{Mol} . \mathrm{NaCl}$
Volumverhältnis $\mathrm{ClO}_{2}: \mathrm{Cl}_{2}$.

$$
\begin{aligned}
& 2: 2.04 \\
& 2: 1.99 \\
& 2: 1.42 \\
& 2: 1.20
\end{aligned}
$$

Er hat den Schlufs gezogen, dafs die Grenzreaktion in sehr verdünnter Chloridlösung folgende sein würde:

$$
2 \mathrm{HClO}_{3}+2 \mathrm{HCl}=2 \mathrm{ClO}_{2}+\mathrm{Cl}_{2}+2 \mathrm{H}_{2} \mathrm{O} .
$$

Die entgegengesetzte Reaktion zwischen Chlor und Chlordioxyd ist oben ( $§$ III, 7) beschrieben worden, so daf's die Möglichkeit eines Gleichgewichtes vorzuliegen schien.

\section{Eigene Versuche.}

Um diese Frage näher zu prüfen, habe ich zunächst zur Ergänzung die Ergebnisse ScHacherLs durch folgende Versuche bestätigt.

1 SchacherL, Lieb. Ann. 182 (1876), 193. 
Bekannte Mengen von $\mathrm{NaCl}, \mathrm{NaClO}_{3}$ und $\mathrm{H}_{2} \mathrm{SO}_{4}$-Lösungen wurden zusammengebracht und die langsam entstehenden Gase $\mathrm{Cl}_{2}$ und $\mathrm{ClO}_{2}$ - mittels eines regelmälsigen Luftstromes in eine neutrale Jodkaliumlösung eingeleitet. Aus dem Verhältnis zwischen den zwei Jodtitrationen (in saurer und in neutraler Lösung) kann man ja den Chlorgehalt des Gases annähernd berechnen, (vergl. §I). Für ein Gas, welches gleiche Verbindungsgewichte von Chlor und $\mathrm{ClO}_{2}$ enthält, müfste das Verhältnis der beiden Jodtitrationen etwa 2.9 betragen.

Um ein Gasgemisch zu erhalten, in dem Chlor und $\mathrm{ClO}_{2}$ im selben Verhältnis vorhanden waren, in dem sie entstehen, um also Unterschiede in der Entweichungsgeschwindigkeit zu vermeiden, wurde der Gasstrom einige Zeit gehen gelassen, eher er in die JKLösung eingeleitet wurde und dann immer mehrere Messungen ohne Zwischenpausen ausgeführt.

Die Resultate einiger Messungen sind in den folgenden zwei Tabellen gegeben. Die "Jodtitration" ist wie immer die gesamte Titration in saurer Lösung. Die Versuche in Tabelle 17 bei $20^{\circ}$ bezw. $\left(50^{\circ}\right.$ und $\left.40^{\circ}\right)$ wurden zu verschiedenen Zeiten angestellt.

Tabelle 17.

$\begin{array}{ll}\text { Chlornatrium } & 0.2 \mathrm{Mol} \text { - Zum Schlufs } 0.076 \mathrm{Mol} \text {. } \\ \mathrm{NaClO}_{3} & 2.0 \mathrm{n} \\ \text { Schwefelsäure } & 4.0 \mathrm{normal} \\ \text { Volum } & 40 \mathrm{ccm}\end{array}$

Temperatur in

$\begin{array}{lrccccccc}\text { Temperatur in } 0 & 20 & 20 & 50 & 45 & 40 & 50 & 50 & 50 \\ \text { Zeit in Minuten } & 210 & 45 & 20 & 60 & 30 & - & - & 30 \\ \text {,Jodtitration" cem n/10 Thios. } & 30.8 & 6.3 & 17.5 & - & 18.7 & 10.6 & 8.85 & 12.05 \\ \text { Verbältnis der zwei Jodtitration } & 2.75 & 2.74 & 2.67 & 2.87 & 2.96 & 2.38 & 2.55 & 2.54\end{array}$

$\begin{array}{lllllllll}\text { Verhältnis der zwei Jodtitration } & 2.75 & 2.74 & 2.67 & 2.87 & 2.96 & 2.38 & 2.55 & 2.54\end{array}$

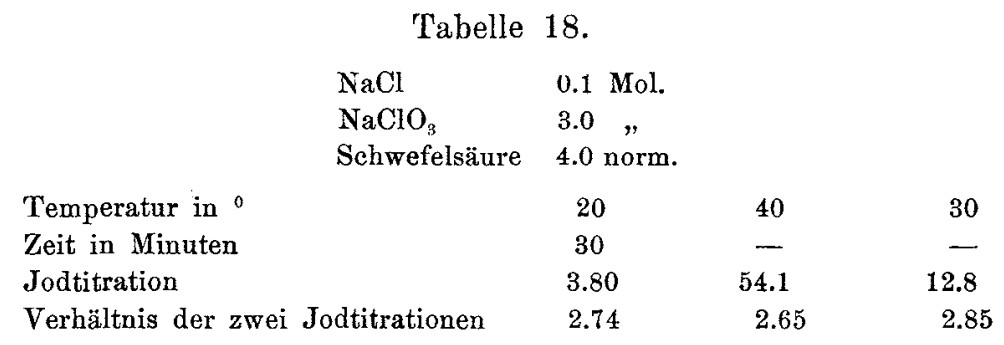

Es folgt hieraus, dafs die „Jodtitration“ etwa 2.7-2.8 mal gröfser ist, als die Jodtitration in neutraler Lösung, d. b. es entsteht 1 Teil 
$\mathrm{ClO}_{2}$ auf etwa 1.1 und 1.2 Teile Chlor $\left({ }^{1} / 2 \mathrm{Cl}_{2}\right)$ (Volumverhältnisse $2: 1.1-2: 1.2)$.

Der Chlorgehalt des Gases nimmt zu mit zunehmender Temperatur (vergl. SchacherL l. c.).

In einem anderen Versuch wurden nicht die Menge der Entstehungsprodukte, sondern die Abnahme der Konzentration der Ausgangsstoffe $\left(\mathrm{Cl}^{\prime}\right.$ - und $\mathrm{H} \cdot$-Ionenkonzentration), bestimmt mit folgendem Ergebnis:

Tabelle 19.

Versuchsdauer

18 Stunden

$\mathrm{NaClO}_{\mathrm{a}}$-Konzentration. $2.5 \mathrm{Mol}$.

$\begin{array}{lll} & \text { vorher } & \text { nachher } \\ \text { Säurekonzentration } & 3.51 & 3.10 \text { norm. } \\ \text { Chlorionkonzentration } & 0.497 & 0.280 \mathrm{Mol} .\end{array}$

Das Verhältnis zwischen den Mengen von $\mathrm{HClO}_{3}$ und $\mathrm{HCl}$, welche reagiert haben, ist

$$
1.12, \text { woraus } \frac{\mathrm{ClO}_{8}}{\mathrm{Cl}}=1: 1.18
$$

Volumverhältnis $2: 1.18$

welches mit obigen Resultaten gut übereinstimmt. Die Übereinstimmung dieser Versuche mit denen von SoHacherr ist ein Beweis für die Anwendbarkeit der gebrauchten Titrationsmethoden.

\section{Kinetische Betrachtungen.}

Es ist in der Gleichung Schacherls $1 \mathrm{ClO}_{2}$ auf $1 / 2 \mathrm{Cl}_{2}$ (nicht $2 \mathrm{ClO}_{2}$ auf $1 \mathrm{Cl}_{3}$ ) geschrieben worden, um den Zusammenhang mit einigen Ergebnissen kinetischer Untersuchung zu gewinnen.

Eigene Versuche ${ }^{1}$ führten zum Resultat, dafs die Zersetzung der Chlorsäure in Cl'-Ionlösung bei Gegenwart von $\mathrm{J}^{\prime}$ nach der Gleichung:

1 Bray, Journ. Phys. Chem. 7 (1903), 112.

Ich möchte diese Gelegenheit benutzen, um einen Druckfehler auf S. 112 zu korrigieren, wodurch die Ableitung der Werte der Konstante $\left(k_{b}\right)$ unklar wird. $k_{b}$ ist aus der Gleichung $R=0.097+0.009$ n. (S. $107 \mathrm{u}$. Tabelle 34) abgeleitet worden; es ist $\frac{d R}{d D}=100 \mathrm{~A} \cdot \mathrm{C}^{2} \cdot k_{b}=0.009$, woraus sich $k_{b}=18 \times 10^{-8}$ ergibt. In einem anderen Versuch wurde $k_{b}=15 \times 10^{-8}$ gefunden. 


$$
\begin{aligned}
\frac{-d\left(\mathrm{ClO}_{3}{ }^{\prime}\right)}{d t} & ={ }_{4} k\left(\mathrm{ClO}_{3}{ }^{\prime}\right)\left(\mathrm{Cl}^{\prime}\right)\left(\mathrm{H}^{\cdot}\right)^{2} \quad \text { verläuft, wo } \\
{ }_{4} k & =\text { etwa } 18.10^{-5} \text { bei } 30^{0} \text { ist. }^{1}
\end{aligned}
$$

Hierdurch ist die linke Seite einer Reaktionsgleichung der Form: $\mathrm{ClO}_{3}{ }^{\prime}+\mathrm{Cl}^{\prime}+2 \mathrm{H}^{\cdot}$ gegeben, was in voller Übereinstimmung mit der Schacheruschen Reaktion, in verdünnter Cl'-Ionlösung

$$
\mathrm{ClO}_{3}{ }^{\prime}+\mathrm{Cl}^{\prime}+2 \mathrm{H} \longrightarrow \mathrm{ClO}_{2}+1 / 2 \mathrm{Cl}_{2}+\mathrm{H}_{2} \mathrm{O}
$$

steht, wenn man sie, wie es oben geschehen, mit halben Koeffizienten schreibt. Ob tatsächlich die rechts stehenden Stoffe die primären Produkte der Umsetzung sind, ist fraglich; vermutlich geht zuerst die Reaktion:

$$
\mathrm{ClO}_{3}{ }^{\prime}+\mathrm{Cl}^{\prime}+2 \mathrm{H}^{\cdot}=\mathrm{HClO}_{2}+\mathrm{HClO} \text { vor sich. }
$$

Nach eigenen Versuchen ist es sehr wahrscheinlich, dals der Vorgang

$$
\mathrm{HClO}_{2}+\mathrm{HClO}=\mathrm{ClO}_{2}+{ }^{1} / 2 \mathrm{Cl}_{2}+\mathrm{H}_{2} \mathrm{O}
$$

sehr rasch verläuft, so dafs die Möglichkeit für die ebengenannte Zwischenreaktion gegeben ist. Als Stütze für sie kann man nur anführen, dals keine Reaktionen mit Sicherheit bekannt sind, in denen $\mathrm{ClO}_{3} \mathrm{H}$ direkt zu $\mathrm{ClO}_{2}$ reduziert, $\mathrm{Cl}^{\prime}$ direkt $\mathrm{zu}^{1} / 2 \mathrm{Cl}_{3}$ oxydiert wird, wohl aber solche, in denen $\mathrm{ClO}_{3} \mathrm{H}$ primär zu $\mathrm{ClO}_{2} \mathrm{H}$ reduziert, ${ }^{2}$ und $\mathrm{Cl}^{\prime}$ primär $\mathrm{zu} \mathrm{ClO}^{\prime}$ oxydiert wird. ${ }^{3}$

Es mufs noch hierzu bemerkt werden, dals in der kinetisch untersuchten Reaktion (bei Anwesenheit von $\mathrm{J}^{\prime}$-Ion und grofser $\mathrm{Cl}^{\prime}$ Konzentration, im Gegensatz zu den verdünnten $\mathrm{Cl}^{\prime}$-Ionlösungen SchacherL s), diese möglichen Primärstoffe $\mathrm{ClO}_{2} \mathrm{H}$ und $\mathrm{ClOH}$ (bezw. $\mathrm{ClO}_{2}$ und $\mathrm{Cl}_{2}$ ) rasch mit Jodion reagieren, so dafs deren Umsetzung als Folgereaktionen angenommen werden dürfen.

Für molare Salzsäure und molare $\mathrm{KClO}_{3}$-Lösung ergab sich die Geschwindigkeit der tetramolekularen Reaktion bei Anwesenheit von $J^{\prime} \mathrm{zu} \frac{-d\left(\mathrm{ClO}_{3}{ }^{\prime}\right)}{d t}=18.10^{-5}$ bei $30^{\circ}$.

${ }^{1}{ }_{4} k=1000 k_{b}=18.10^{-5}$.

2 Vergl. \& VI.

${ }^{3}$ Zeitschr. phys. Chem. 54 (1906), 463. 
Zur Berechnung der Geschwindigkeit der SchacherLschen Reaktion können die Versuche in Tabelle 17 benutzt werden; diese sind als Geschwindigkeitsversuche wenig genau, erlauben aber einen Schlufs auf die Grölsenordnung. Unter der Annahme, dafs der Vorgang tetramolekular ist, ergibt sich für eine molare HCl- und $\mathrm{KClO}_{3}$-Lösung:

$$
\begin{aligned}
\frac{-d\left(\mathrm{ClO}_{3}{ }^{\prime}\right)}{d t}= & \text { etwa } 1 \cdot 10^{-5} \text { bei } 20^{\circ} \text { und } \\
10 \cdot 10^{-5} \text { bei } 50^{\circ} \text {, woraus sich } & \text { etwa } 2 \cdot 10^{-5} \text { für } 30^{\circ} \text { berechnet. }
\end{aligned}
$$

Wie man sieht, sind beide Geschwindigkeiten von gleicher Grölsenordnung.

\section{Reaktion zwischen Chlorsäure und konzentrierter Salzsäure.}

Der Umstand, dals der $\mathrm{ClO}_{2}$-Gehalt, sowohl bei steigender Temperatur, wie bei steigender $\mathrm{Cl}^{\prime}-$ Ionkonzentration abnimmt, machte es notwendig, die Reaktion zwischen Chlorsäure und konzentrierter Salzsäure besonders zu betrachten.

Sie soll quantitativ nach dem Schema

$$
\mathrm{HClO}_{3}+5 \mathrm{Cl}_{2}=3 \mathrm{Cl}_{2}+3 \mathrm{H}_{2} \mathrm{O}
$$

erfolgen, wie schon oft angenommen worden ist. Da ich keine Angabe darüber fand, ob tatsächlich kein $\mathrm{ClO}_{2}$ entsteht, habe ich folgende Versuche angestellt.

Einige Lösungen wurden in zugeschmolzenen Röhren im Dunkeln bei $60^{\circ}$ erwärmt; nach Verdünnung wurde das entstandene Gas mittels eines Sauerstoffstromes in neutrale KJ-Lösung eingeleitet. (Vergl. \& I.)

Für eine Lösung, die in bezug auf Schwefelsäure normal, in bezug auf Chlornatrium, Natriumchlorat molar war, wurde das oft genannte Verbältnis der zwei Jodtitrationen zu 0.001 gefunden; das bedeutet 1 Teil $\mathrm{ClO}_{2}$ auf etwa 4000 Verbindungsgewichte Chlor (oder 2000 Volumina). Andere Lösungen gaben ähnliche Resultate. Die Lösungen bei $60^{\circ}$ war farblos. Nach längerem Stehen bei Zimmertemperatur wurden sie gelb und die Analysen ergaben die Anwesenheit von $\mathrm{ClO}_{2}$, welches bei $60^{\circ}$ wieder verschwand. 
Die Geschwindigkeit dieser Reaktion ist kürzlich von $\mathbf{S}_{\mathbf{A N D}}{ }^{1}$ gemessen worden; er gelangte zur kinetischen Gleichung:

$$
\frac{1}{3} \frac{d\left(\mathrm{Cl}_{2}\right)}{d t}=\frac{-d\left(\mathrm{ClO}_{8}{ }^{\prime}\right)}{d t}={ }_{5} l\left(\mathrm{ClO}_{3}{ }^{\prime}\right)\left(\mathrm{Cl}^{\prime}\right)^{2}\left(\mathrm{H}^{\cdot}\right)^{2} .
$$

\section{§ VI. Schlufserörterung.}

Obwohl auf Grund von IV u.V eine definitive Antwort auf die Frage nach der primären Reduktion der Oxyhalogensäuren vom Typus $\mathrm{HXO}_{3}$ noch nicht gegeben werden kann, mögen die bisher gewonnenen Resultate hier zusammengestellt werden.

Für einige Reaktionen vom Typus $\mathrm{HXO}_{3}+\mathrm{HY}$ gilt die folgende kinetische Gleichung:

$$
\frac{-d\left(\mathrm{XO}_{3}{ }^{\prime}\right)}{d t}={ }_{4} k\left(\mathrm{XO}_{3}{ }^{\prime}\right)\left(\mathrm{Y}^{\prime}\right)(\mathrm{H} \cdot)^{2}
$$

nämlich für die Reaktionen $\mathrm{HBrO}_{3}+\mathrm{HJ} ;^{2} \mathrm{HBrO}_{3}+\mathrm{HBr} ;{ }^{3} \mathrm{HClO}_{3}$ $+\mathrm{HJ}^{4}$ und $\mathrm{HClO}_{3}+\mathrm{HCl}^{4}$ (die letzteren beiden nebeneinander).

Die Theorie, dals die $\mathrm{HXO}_{3}$-Säure $\mathrm{zu} \mathrm{HXO}_{2}$ reduziert wird und dafs HY zu der HYO-Stufe oxydiert wird, wurde von Noxes vorgeschlagen und von den anderen Forschern angenommen. Man kann die folgende primäre Gleichung aufstellen:

oder

$$
\mathrm{XO}_{3}{ }^{\prime}+\mathrm{Y}^{\prime}+2 \mathrm{H}^{\circ}
$$

$$
\mathrm{HXO}_{3}+\mathrm{HY}=\mathrm{HXO}_{2}+\mathrm{HYO} \text {. }
$$

Diese etwas willkürliche Annahme wird durch folgende Resultate unterstützt:

a) Wie in einer anderen Abhandlung ${ }^{5}$ ausgeführt wurde, ist

${ }^{1} \mathrm{~S}_{\mathrm{AND}}$, Zeitschr. phys. Chem. 50 (1904), 465.

Es sind indes eine Reihe berechtigter Zweifel an der Richtigkeit der SANDschen Auffassung aufgestiegen (Ordnung bezüglich $\mathrm{ClO}_{3}{ }^{\prime}$ u. a.), die nur durch den Versuch geklärt werden können. Deshalb sollen seine Ergebnisse im Vergleich zu den meinen bezw. denen SchacherL 8 vorerst noch nicht erörtert werden. Siehe aber den Schlufs dieser Abhandlung.

${ }^{2}$ Nores, Zeitschr. phys. Chem. 18 (1895), 118 und 19 (1896), 599; vergl. Zeitschr. phys. Chem. 54 (1906), 467.

s Judson u. Walker, Journ. Chem. Soc. 73 (1898), 410, Trans.

${ }^{4}$ Bnax, Journ. phys. Chem. 7 (1903), 92.

5 Zeitschr. phys. Chem. 54 (1906), 463. 
eine primäre Oxydation von $\mathrm{Y}^{\prime}$ zu der HYO-Stufe in vielen anderen Reaktionen äufserst wahrscheinlich;

b) eine primäre Reduktion von Chlorsäure zu chloriger Säure ist die einfachste Erklärung für die von NoYES und WASON ${ }^{1}$ untersuchte trimolekulare Reaktion zwischen $\mathrm{Fe} \cdot, \mathrm{ClO}_{3}{ }^{\prime}$ und $\mathrm{H}^{\circ}$-Ionen.

Die Verfasser hielten eine solche Reaktion für wahrscheinlich, sagten aber, da sie keinen einfachen Mechanismus gefunden hätten, „dafs die Reaktion wenigstens anscheinend eine Ausnahme von VAN'T HoFrs Grundsatz bildet".. ${ }^{2}$ Wenn man aber annimmt, dals in dieser Reaktion $\mathrm{Fe}^{*}$ nicht direkt zu Fe"*, sondern wie in vielen anderen Reaktionen ${ }^{3}$ zu einem „Peroxyd" oxydiert wird, dann bekommt man folgende einfache primäre Gleichung:

oder

$$
\mathrm{Fe} \cdot+\mathrm{ClO}_{3}{ }^{\prime}+\mathrm{H} \cdot
$$

$$
\mathrm{Fe}^{*}+\mathrm{HClO}_{3}=\mathrm{FeO}^{*}+\mathrm{HClO}_{2}
$$

und die "momentan verlaufenden“ Folgereaktionen.

$$
\begin{gathered}
\mathrm{Fe}^{*}+\mathrm{FeO}^{*}+2 \mathrm{H}^{*}=2 \mathrm{Fe}^{\cdots}+\mathrm{H}_{2} \mathrm{O} \\
4 \mathrm{Fe}^{*}+\mathrm{HClO}_{2}+3 \mathrm{H}^{*}=4 \mathrm{Fe}^{\cdots}+\mathrm{Cl}^{\prime}+2 \mathrm{H}_{2} \mathrm{O},
\end{gathered}
$$

welche zusammen die Bruttogleichung:

$$
6 \mathrm{Fe}^{*}+\mathrm{ClO}_{3}{ }^{\prime}+6 \mathrm{H}^{\cdot}=6 \mathrm{Fe}^{\cdots}+\mathrm{Cl}^{\prime}+3 \mathrm{H}_{2} \mathrm{O}
$$

ergeben.

Die Folgereaktion (a) ist schon öfters angenommen worden; die Reaktion (b) erwies sich als eine momentan verlaufende. Folglich ist die primäre Gleichung eine mögliche und auch eine wahrscheinliche Erklärung der kinetischen Resultate. Die Annahme einer primären Reduktion zu $\mathrm{ClO}_{2}$ ist nicht möglich.

c) Nach Lother und Sontow (l. c.) sind, wie öfters erwähnt, die bei der Reduktion von Bromsäure beobachteten „Koppelungs-

1 Noyes u. Wason, Zeitschr. phys. Chem. 22 (1897), 210.

2 Ostwald, Lehrbuch II, 2, S. 243, erörtert die Möglichkeit einer Reaktion $\mathrm{Fe}^{*}+\mathrm{ClO}_{3}{ }^{\prime}+\mathrm{H}^{*}=\mathrm{Fe}^{\cdots}+\mathrm{HClO}_{3}{ }^{\prime}$, hält aber die Annahme einer solchen hypothetischen Oxychlorsäure für sehr willkürlich.

${ }^{3}$ Manchot u. Zimmermans, Lieb. Ann. 325 (1902), 105. - Luther und Sonixow, Zeitschr. phys. Chem. 46 (1903), 777. - Schluow, Zeitschr. phys. Chem. 42 (1903), 641.

4 Eventuell kann Gleichung (b) wieder zerlegt werden. 
erscheinungen" quantitativ durch die Annahme einer primären $\mathrm{HBrO}_{2}$-Bildung erklärt, während die Annahme einer $\mathrm{BrO}_{2}$-Bildung nicht zum Ziel führt. Und bezüglich der Chlorsäure haben qualitative Versuche der ebengenannten Autoren und anderen es jedenfalls wahrscheinlich gemacht, dafs chlorige Säure als Zwischenstufe bei den entsprechenden Koppelungserscheinungen eine Rolle spielt.

Jedenfalls erscheint es vorläufig als das wahrscheinlichste bei der Reduktion von Brom- und Chlorsäure, bromige Säure, bezw.

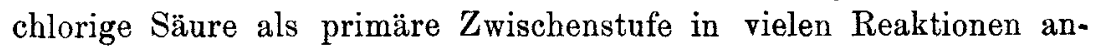
zunehmen.

Die Existenz des Gleichgewichtes:

$$
\mathrm{ClO}_{3}{ }^{\prime}+\mathrm{Cl}^{\prime}+2 \mathrm{H} \cdot \rightleftarrows \mathrm{ClO}_{2}+{ }^{1} /{ }_{2} \mathrm{Cl}_{2}+\mathrm{H}_{2} \mathrm{O}
$$

weist jedenfalls darauf hin, dafs die Annahme der tetramolekularen Reaktion

$$
\mathrm{ClO}_{3}{ }^{\prime}+\mathrm{Cl}^{\prime}+2 \mathrm{H} \cdot=\mathrm{ClO}_{2} \mathrm{H}+\mathrm{ClOH}=\mathrm{ClO}_{2}+{ }^{1 / 2}{ }_{2} \mathrm{Cl}_{2}+\mathrm{H}_{2} \mathrm{O}
$$

als reeller, primärer Reaktion bei kleiner $\mathrm{Cl}^{\prime}$-Ionkonzentration und bei Gegenwart ron $J^{\prime}$-Ion durchaus wahrscheinlich ist. Wie allerdings der Übergang von dieser tetramolekularen Reaktion zu der ,pentamolekularen“ Reaktion SANDs (bei grofser Cl'-Ionkonzentration) zu denken ist, läfst sich, wie schon in $\S \mathrm{V}$ erwähnt wurde, vorerst nicht entscheiden. An der gleichen Stelle ist auch bemerkt worden, dafs ernstliche Zweifel an den pentamolekularen Verlauf dieser Reaktion aufgestiegen sind; denn eine Durchrechnung der SANDschen Zahlen ergab, worauf mich Prof. Luther aufmerksam machte, mit ziemlicher Sicherheit einen tetramolekularen Verlauf nach der sehr eigentümlichen Gleichung:

$$
\frac{1}{3} \frac{d\left(\mathrm{Cl}_{2}\right)}{d t}=-\frac{d\left(\mathrm{ClO}_{3}{ }^{\prime}\right)}{d t}={ }_{4} k\left(\mathrm{Cl}_{2}{ }^{\prime}\right)^{2} \cdot\left(\mathrm{H}^{\cdot}\right)^{2} .
$$

\section{Zusammenfassung.}

1. Die Zersetzung von $\mathrm{ClO}_{2}$ unter verschiedenen Umständen und seine Reaktion mit $\mathrm{OH}^{\prime}$ wurde untersucht und erörtert.

2. Es wurde gezeigt, dals das oft angenommene hydrolytische Gleichgewicht:

$$
2 \mathrm{ClO}_{2}+\mathrm{H}_{2} \mathrm{O} \longleftrightarrow \mathrm{HClO}_{3}+\mathrm{HClO}_{2}
$$

nicht die vermutete Bedeutung besitzt. 
3. Es konnte wahrscheinlich gemacht werden, dals die Reduktion der $\mathrm{ClO}_{3} \mathrm{H}$ mit primärer Bildung der Chloritstufe erfolgt. Jedenfalls existiert bei kleiner $\mathrm{Cl}^{\prime}$-Konzentration das Gleichgewicht:

$$
2 \mathrm{H} \cdot+\mathrm{ClO}_{3}{ }^{\prime}+\mathrm{Cl}^{\prime} \longleftrightarrow \mathrm{ClO}_{2}+1 / 2 \mathrm{Cl}_{2}+\mathrm{H}_{2} \mathrm{O} \text {. }
$$

Diese Arbeit wurde im phys.-chem. Institut zu Leipzig unter der Leitung von Prof. R. LuTHER ausgeführt.

Leipzig, Physik.-ehem. Institut der Universität.

Bei der Redaktion eingegangen am 19. Dezember 1905. 